\title{
Statistical Concurrent Non-malleable Zero-knowledge from One-way Functions
}

\author{
Susumu Kiyoshima \\ NTT Secure Platform Laboratories, Japan \\ kiyoshima.susumu@lab.ntt.co.jp
}

December 18, 2015

\begin{abstract}
Concurrent non-malleable zero-knowledge (CNMZK) protocols are zero-knowledge protocols that provides security even when adversaries interacts with multiple provers and verifiers simultaneously. It is known that CNMZK arguments for $\mathcal{N P}$ can be constructed in the plain model. Furthermore, it was recently shown that statistical CNMZK arguments for $\mathcal{N} \mathcal{P}$ can also be constructed in the plain model. However, although the former requires only the existence of one-way functions, the latter requires the DDH assumption.

In this paper, we construct a statistical CNMZK argument for $\mathcal{N} \mathcal{P}$ assuming only the existence of one-way functions. The security is proven via black-box simulation, and the round complexity is $\operatorname{poly}(n)$. Under the existence of collision-resistant hash functions, the round complexity is reduced to $\omega(\log n)$, which is essentially optimal for black-box concurrent zero-knowledge protocols.
\end{abstract}

This article is based on an earlier article: Statistical Concurrent Non-malleable Zero-knowledge from One-way Functions, in Proceedings of CRYPTO 2015, (CIACR 2015, DOI: 10.1007/978-3-662-48000-7_5. 


\section{Introduction}

Zero-knowledge (ZK) proofs and arguments are protocols that enable the prover to convince the verifier of the correctness of a mathematical statement while providing zero additional knowledge. This "zero additional knowledge" property is formalized by using the simulation paradigm: An interactive proof or argument is said to be zero-knowledge if for any adversarial verifier there exists a simulator that can output a simulated view of the adversary. In the original definition of the ZK property, the adversary interacts with a single prover at a time. In other words, in the original definition, the ZK property is considered in the stand-alone setting.

Non-malleable zero-knowledge (NMZK) [DDN00] and concurrent zero-knowledge (CZK) [DNS04] are two well-known notions of the ZK property in the concurrent setting. Specifically, NMZK is a notion of the ZK property in the setting where the adversary concurrently interacts with a honest prover in the left session and a honest verifier in the right session, and CZK is a notion of the ZK property in the setting where the adversary concurrently interacts with unbounded number of honest provers.

As a security notion that implies both NMZK and CZK, Barak et al. [BPS06] proposed concurrent non-malleable zero-knowledge (CNMZK). CNMZK guarantees the ZK property in the setting where the adversary concurrently interacts with many provers in the left sessions and many verifiers in the right sessions. In particular, it guarantees that receiving proofs in the left session does not help the adversary to give proofs in the right sessions-that is, it guarantees that if the adversary can prove some statements in the right sessions while receiving proofs in the left sessions, the adversary could prove the same statements even without receiving proofs in the left sessions. In the definition of CNMZK, this guarantee is formalized as the existence of a simulator-extractor that can simulate the adversary's view in the left and right sessions while extracting witnesses from the adversary in the simulated right sessions.

The first CNMZK argument for $\mathcal{N} \mathcal{P}$ was constructed by Barak et al. [BPS06]. Subsequently, a computationally efficient construction was shown by Ostrovsky et al. [OPV10]. The first CNMZK proof was constructed by Lin et al. [LPTV10], and a variant of their protocol was shown to be secure with adaptively chosen inputs by Lin and Pass [LP11a]. Additionally, a CNMZK argument that is secure with "fully" adaptively chosen inputs was recently constructed by Venkitasubramaniam [Ven14].

Very recently, Orlandi et al. [OOR $\left.{ }^{+} 14\right]$ constructed the first statistical CNMZK argument, i.e., a CNMZK argument such that the simulator-extractor outputs view that is statistically indistinguishable from the adversary's real view. Statistical CNMZK is clearly of great interest since it guarantees quite strong security in the concurrent setting. However, statistical CNMZK is hard to achieve, and the existing techniques of computational CNMZK protocols seem to be insufficient for constructing statistical CNMZK protocols (see Section 2.1).

An important open question on statistical CNMZK protocols is what hardness assumption is needed for constructing them. The statistical CNMZK argument of Orlandi et al. [OOR $\left.{ }^{+} 14\right]$ was constructed under the DDH assumption (or the existence of dense cryptosystems). Hence, we already know that statistical CNMZK protocols can be constructed under standard assumptions. However, since the existence of one-way functions is known to be sufficient for constructing both statistical ZK protocols and computational CNMZK protocols [ $\mathrm{HNO}^{+}$09, BPS06], it is important to study the following question.

Can we construct statistical concurrent non-malleable zero-knowledge protocols by assuming only the existence of one-way functions? 


\subsection{Our Result}

In this paper, we answer the above question affirmatively.

Theorem 1. Assume the existence of one-way functions. Then, there exists a statistical concurrent non-malleable zero-knowledge argument for $\mathcal{N P}$ with round complexity poly $(n)$. Furthermore, if there exists a family of collision-resistant hash functions, the round complexity can be reduced to $\omega(\log n)$.

The round complexity of our statistical CNMZK argument-poly $(n)$ rounds when only the existence of one-way functions is assumed and $\omega(\log n)$ rounds when the existence of a family of collision-resistant hash functions is assumed-is the same as the round complexity of the known statistical CZK arguments [GMOS07]. Thus, our result closes the gap between statistical CNMZK arguments and statistical CZK arguments. Furthermore, since the security of our statistical CNMZK protocol is proven via black-box simulation, the logarithmic round complexity of our hash-functionbased protocol is essentially tight due to the lower bound on black-box CZK protocols [CKPR02].

\section{Techniques}

In this section, we give an overview of our techniques.

\subsection{Previous Techniques}

We start by describing the difficulty of constructing statistical CNMZK protocols with the techniques of existing computational CNMZK protocols [BPS06, LPTV10].

First, let us recall the protocols of [BPS06, LPTV10]. The definition of CNMZK requires the existence of a simulator-extractor that simulates the adversary's view while extracting the witnesses for the statements proven by the adversary in the simulated view. To satisfy this definition, protocols need to satisfy the following properties: (i) the proofs in the left sessions can be simulated for the adversary, and (ii) even when the adversary receives simulated proofs in the left sessions, the witnesses can be extracted from the adversary in the right sessions. In the protocol of [BPS06, LPTV10], the simulatability of the left sessions is guaranteed by requiring the verifier to commit to a random trapdoor by using a concurrently extractable commitment scheme CECom [MOSV06]. The committed values of CECom can be extracted by a rewinding extractor even in the concurrent setting, and therefore the proofs in the left sessions can be simulated by extracting the trapdoors from CECom. On the other hand, the witness-extractability of the right sessions is guaranteed by requiring the prover to commit to the witness with a non-malleable commitment scheme NMCom [DDN00] and additionally designing the protocols so that the following hold.

1. When the adversary receives honest proofs in the left sessions, the committed value of the NMCom commitment is indeed a valid witness in every accepted right session.

2. When the proofs in the left sessions are switched to the simulated ones, the committed values of the NMCom commitments do not change in the right sessions due to the non-malleability of NMCom.

It follows from these that even when the adversary receives simulated proofs in the left sessions, the committed value of the NMCom commitment is a witness for the statement in every accepted right session. Therefore, the witnesses can be extracted in the right sessions by extracting the committed values of the NMCom commitments. 
As mentioned in Introduction, the techniques of [BPS06, LPTV10] alone seem to be insufficient for constructing statistical CNMZK protocols. The main obstacle is that the techniques of [BPS06, LPTV10] requires the prover to commit to the witness by using NMCom, which is only computationally hiding. ${ }^{1}$ Since the committed values of NMCom in the left sessions need to be switched to another values (e.g., $0^{n}$ ) in the simulation, the simulated view can be only computational indistinguishable from the real view.

Recently, Orlandi et al. [OOR $\left.{ }^{+} 14\right]$ constructed a statistical CNMZK protocol by modifying the CNMZK protocol of [BPS06] with mixed non-malleable commitment scheme MXNMCom. MXNMCom is parametrized by a string and is either statistically hiding or non-malleable depending on the string. ${ }^{2}$ Very roughly speaking, Orlandi et al. circumvent the above problem by carefully switching the parameter string of MXNMCom in the security proof-when proving the statistical indistinguishability of the simulation, the string is set so that MXNMCom is statistically hiding, and when proving the non-malleability, the string is set so that MXNMCom is non-malleable. The use of MXNMCom, however, requires assumptions that are seemingly stronger than the existence of one-way functions (such as the DDH assumption or the existence of dense cryptosytems). Thus, the technique of Orlandi et al. cannot be used to construct statistical CNMZK protocols from one-way functions.

\subsection{Our Technique}

Since the reason why the techniques of [BPS06, LPTV10] cannot be used for statistical CNMZK protocols is that the committed values of NMCom need to be switched during the simulation, one potential strategy for constructing statistical CNMZK is to construct a protocol such that the adversary's view can be simulated without switching the committed value of NMCom (and of any other computationally hiding commitment). However, when the simulator commits to the same value in NMCom as a honest prover, it is not clear how non-malleability of NMCom can be used in the security proof. Below, we show that the CNMZK property can be shown even in this case if we use a stronger variant of NMCom.

A key technical tool in our technique is CCA-secure commitment schemes [CLP10], which is a stronger variant of (concurrent) non-malleable commitment schemes. Roughly speaking, CCA security guarantees that the scheme is hiding even against adversaries that have access to the committedvalue oracle, which receives concurrent commitments from the adversary and returns their committed values to the adversary. (In non-malleability, the oracle receives only parallel commitments from the adversary and returns the committed values only after the adversary finishes the interaction with the committer.) Several CCA-secure commitment schemes were constructed from one-way functions [CLP10, LP12, Kiy14, GLP $\left.{ }^{+} 15\right]$; furthermore, although CCA security itself does not provide any extractability, all of these schemes satisfy concurrent extractability as well.

Using CCA-secure commitment schemes, we consider the following protocol as a starting point.

\section{Stage 1. ( $V$ commits to trapdoor)}

1. The verifier $V$ chooses random $r_{V} \in\{0,1\}^{n}$ and commits to $r_{V}$ by using a statistically binding commitment scheme Com, which can be constructed from one-way functions [Nao91, HILL99]. Let $\left(r_{V}, d\right)$ be the decommitment.

\footnotetext{
${ }^{1} \mathrm{NMCom}$ need to be non-malleable w.r.t. commitment [DDN00], which roughly says that the committed value of the commitment that the man-in-the-middle adversary gives is independent of the committed value of the commitment that adversary receives. Since the definition of non-malleability w.r.t. commitment is meaningless when the committed values cannot be uniquely determined, NMCom cannot be statistically hiding.

${ }^{2}$ Specifically, Orlandi et al. [OOR $\left.{ }^{+} 14\right]$ used the scheme such that (i) when the string is sampled from a uniform distribution, the scheme is statistically hiding and (ii) when the string is taken from another (computationally indistinguishable) distribution, the scheme is non-malleable.
} 
2. $V$ commits to $\left(r_{V}, d\right)$ by using CCA-CECom, where CCA-CECom is a CCA-secure commitment scheme that is also concurrent extractable [CLP10, LP12, Kiy14, GLP $\left.{ }^{+} 15\right]$.

Stage 2. ( $P$ proves $x \in L$ or knowledge of trapdoor) The prover $P$ proves that it knows a witness for $x \in L$ or a valid decommitment $\left(r_{V}, d\right)$ of the Com commitment that $V$ gives in Stage 1. $P$ proves this statement by using a statistical witness-indistinguishable argument of knowledge sWIAOK, which can be constructed from one-way functions by instantiating Blum's Hamiltonian-cycle protocol with the statistically hiding commitment scheme of $\left[\mathrm{HNO}^{+} 09\right]$.

In this protocol, the verifier's view can be statistically simulated by a simulator that extracts $\left(r_{V}, d\right)$ from CCA-CECom and uses it as a witness in SWIAOK. (During the extraction in Stage 1, the simulator interacts with the verifier honestly; thus, even if computationally hiding commitment schemes are used as building blocks in CCA-CECom, the simulator commits to the same values as a honest prover in these schemes.) Also, intuitively this protocol seems to be CNMZK from the following reasons.

- The CCA security of CCA-CECom guarantees that the trapdoors of the right sessions are hidden from the adversary even when the trapdoors of the left sessions are extracted from the adversary.

- Then, since the simulated proofs are generated in the left sessions by extracting the trapdoors of the left sessions, the trapdoors in the right sessions are hidden from the adversary even when the adversary receives simulated proofs in the left sessions.

- Thus, even when the adversary receives the simulated proofs in the left sessions, the adversary cannot "cheat" in the right sessions, and therefore witnesses for the statements must be extractable from sWIAOK in the right sessions.

Of course, to formally prove the statistical CNMZK property, we need to show a simulator-extractor that statistically simulates the adversary's view and also extracts witnesses for the statements in the right sessions.

As the simulator-extractor, we consider the following $\mathcal{S E}$.

1. First, $\mathcal{S} \mathcal{E}$ simulates the view of the adversary $\mathcal{A}$ by executing the following simulator $\mathcal{S}$ : Simulator $\mathcal{S}$ internally invokes $\mathcal{A}$ and interacts with it in the left and right sessions honestly as provers and verifiers except that in each left session, $\mathcal{S}$ extracts $\left(r_{V}, d\right)$ by using the concurrent extractor of CCA-CECom and uses it as a witness in sWIAOK.

2. After simulating the view of $\mathcal{A}$ as above, $\mathcal{S} \mathcal{E}$ extracts witnesses from the right sessions by doing the following for each right session. First, $\mathcal{S} \mathcal{E}$ rewinds $\mathcal{S}$ until the point just before $\mathcal{S}$ sends the challenge message of sWIAOK to $\mathcal{A}{ }^{3}$ Then, $\mathcal{S} \mathcal{E}$ repeatedly executes $\mathcal{S}$ from this point with flesh randomness until it obtains another accepted transcript of sWIAOK. After obtaining another accepted transcript, $\mathcal{S} \mathcal{E}$ extracts a witness by using the argument-of-knowledge property of sWIAOK.

It is not hard to see that $\mathcal{S E}$ statistically simulates the real view of $\mathcal{A} .{ }^{4}$ Thus, it remains to show that $\mathcal{S E}$ extracts witnesses for the statements in the right sessions.

To show the witness extractability of $\mathcal{S E}$, a natural approach is to follow the above-mentioned approach of [BPS06, LPTV10] and show the following.

\footnotetext{
${ }^{3}$ Since $\mathcal{S}$ rewinds $\mathcal{A}$ during the concurrent extraction of CCA-CECom, $\mathcal{S}$ may send the challenge message of sWIAOK of a right session to $\mathcal{A}$ multiple times. Here, $\mathcal{S} \mathcal{E}$ rewinds $\mathcal{S}$ until the point just before $\mathcal{S}$ sends it to $\mathcal{A}$ on the "main thread."

${ }^{4}$ Formally, we need to show that in the CCA-CECom commitment of the left session, $\mathcal{A}$ commits to a valid decommitment of the Com commitment except with negligible probability. In this overview, however, we ignore this issue for simplicity. For details, see the formal proof in Section 4.2.
} 
1. When $\mathcal{A}$ receives honest proofs in the left sessions, a witness for the statement is extracted from the sWIAOK proof in every accepted right session.

2. When the honest proofs in the left sessions are switched to the simulated ones, the value extracted from sWIAOK does not change in every accepted right session.

Note that here we argue about the extracted values instead of the committed values. At first sight, it seems that this is not a big difference and therefore it seems that the above can be shown by using an argument similar to the one used in [BPS06, LPTV10].

However, this approach does not work. In particular, we do not know how to prove the second part-that is, we cannot show that the extracted values remain to be the same when the honest proofs in the left sessions are switched to the simulated ones. To see this, observe the following. Since the witnesses used in SWIAOK are switched in the simulated proofs, we need to use the witness indistinguishability of sWIAOK of the left sessions to show the indistinguishability of the extracted values. However, since $\mathcal{A}$ is rewound during the witness extraction of the sWIAOK proofs of the right sessions, if the left and the right sessions are scheduled so that the sWIAOK proofs of the left sessions are executed in parallel with the sWIAOK proofs of the right sessions, the sWIAOK proofs of the left sessions are also rewound, and thus we cannot use their witness indistinguishability. ${ }^{5}$

Thus, we instead use the following approach. Informally, the above approach does not work because the honest proofs and the simulated proofs are "too different." We thus introduce a hybrid experiment in which $\mathcal{A}$ receives hybrid proofs in the left sessions, where a hybrid proof is generated by extracting $\left(r_{V}, d\right)$ by brute force and using it as a witness in sWIAOK. (Notice that the only difference between the hybrid proofs and the simulated proofs is how the trapdoors are extracted.) We then show that (i) witnesses for the statements are extracted in the right sessions when $\mathcal{A}$ receives hybrid proofs in the left sessions, and (ii) when hybrid proofs are switched to the simulated ones, the extracted values do not change. More precisely, our analysis proceeds as follows.

- First, we show the second part, i.e., we show that the values extracted in the right sessions do not change when the proofs in the left sessions are switched from the hybrid proofs to the simulated ones. Since the only difference between the hybrid proofs and the simulated ones is how the committed values of the CCA-CECom commitments are extracted (by brute-force or by the concurrent extractability), we can show this by using the concurrent extractability of CCA-CECom. We note however that there is a subtlety since CCA-CECom in the left sessions can be rewound not only by the concurrent extractor of CCA-CECom but also by the extractor of sWIAOK. Nonetheless, by carefully using a standard technique (the "good prefix" argument), we can show that the concurrent extractor of CCA-CECom works even in this case.

- Next, we show that in the hybrid experiment, witnesses for the statements are extracted from the right sessions. Since the simulated proofs can be efficiently generated given access to the committed-value oracle of CCA-CECom, at first sight it seems that this follows directly from the CCA security of CCA-CECom and argument-of-knowledge property of sWIAOK-if a witness for the statement is not extracted, $\left(r_{V}, d\right)$ must be extracted, and thus we can break the CCA security of CCA-CECom. However, there are two problems.

1. Since CCA-CECom in the left sessions can be rewound during the witness extraction of sWIAOK of the right sessions, the hybrid experiment cannot be emulated even given

\footnotetext{
${ }^{5}$ If we use the robust extraction technique [GLP $\left.{ }^{+} 15\right]$, for each left session there exists a rewinding strategy that allows us to extract witnesses from the right sessions without rewinding sWIAOK of this left session. However, since what we want to show is that the values extracted in the right sessions by the rewinding strategy that $\mathcal{S} \mathcal{E}$ uses are unchanged, the robust extraction technique cannot be used here (unless there exists a rewinding strategy that allows us to extract witnesses from the right sessions without rewinding the sWIAOK proof of every left session).
} 
access to the committed-value oracle of CCA-CECom. Hence, the CCA-secure commitments in the right sessions may not be hiding in the hybrid experiment.

2. Since the adversary obtains hybrid proofs, which are generated in super-polynomial time, the argument-of-knowledge property of sWIAOK may not hold in the hybrid experiment. We remark that although existing CCA-secure commitment schemes provides robustness, which guarantees that arbitrary "small"-round protocol remains secure even when adversaries have access to the committed-value oracle, we cannot use robustness here since CCA-CECom in the left sessions can be rewound during the witness extraction of sWIAOK of the right sessions and therefore the hybrid experiment cannot be emulated even given access to the committed-value oracle.

Because of these problems, we cannot use the security of CCA-CECom directly in the analysis. Thus, instead of using existing CCA-secure commitment schemes in a modular way, we directly use their building blocks in the protocol and directly use their proof technique in the analysis. (In particular, we use the robust concurrent extraction technique of $\left[\mathrm{GLP}^{+} 15\right]$ and a one-one CCA-secure commitment scheme of [KMO14].) The proof techniques of existing CCA-secure commitment schemes are strong enough to solve the above problems, and thus we can show that witnesses for the statements are extracted in the hybrid experiment.

From the above two, it follows that even when $\mathcal{A}$ receives simulated proofs in the left session, valid witnesses are extracted in right sessions. This completes the overview of our techniques.

\section{Preliminaries}

\subsection{Notations}

We use $n$ to denote the security parameter. For any $k \in \mathbb{N}$, let $[k] \stackrel{\text { def }}{=}\{1, \ldots, k\}$. For any two-party protocol $\langle A, B\rangle$, we use view $B[A(x) \leftrightarrow B(y)]$ to denote a random variable representing the view of $B$ in the interaction between $A$ and $B$ with input $x$ and $y$ respectively, and use output ${ }_{A, B}[A(x) \leftrightarrow B(y)]$ (resp., output ${ }_{B}[A(x) \leftrightarrow B(y)]$ ) to denote a random variable representing the joint output of $A$ and $B$ (resp., the output of $B$ ) in the interaction between $A$ and $B$ with input $x$ and $y$ respectively.

\subsection{Commitment Schemes}

Recall that commitment schemes are two-party protocols between the committer $C$ and the receiver $R$. A transcript of the commit phase is valid if there exists a valid decommitment of this transcript.

It is known that a two-round statistically binding commitment scheme $\mathrm{Com}_{\mathrm{SB}}$ can be constructed from one-way functions [Nao91, HILL99]. It is also known that a poly(n)-round statistically hiding commitment scheme can be constructed from one-way functions $\left[\mathrm{HNO}^{+} 09\right]$ and a constant-round one can be constructed from a family of collision-resistant hash functions [NY89, DPP98].

\subsection{Concurrently Extractable Commitment Schemes}

Roughly speaking, a commitment scheme is concurrently extractable if there exists a polynomialtime extractor such that for any adversarial committer that concurrently commits to many values by using the scheme, the extractor can extract the committed value from the adversarial committer in every valid commitment.

Micciancio et al. [MOSV06] proposed a $\omega(\log n)$-round concurrently extractable commitment CECom (Figure 1), which is an abstraction of the preamble stage of the concurrent zero-knowledge 
protocol of [PRS02] and can be constructed from one-way functions. The extractor of CECom performs the extraction by rewinding the adversarial committer according to the rewinding strategy of [PRS02, PTV12]. Specifically, while interacting with the adversarial committer $C^{*}$ on the "main thread" as honest receivers, the extractor rewinds the main thread and generates many "look-ahead threads" on which it interacts with $C^{*}$ again as honest receivers with flesh randomness; at the end of each commitment on each thread, the extractor extracts the committed values by using the information collected on the other threads.

CECom can be seen as a concurrent execution of the extractable commitment scheme ExtCom in Figure 2, which consists of three stages—commit, challenge, and reply—and can be constructed from one-way functions.

\section{Commit Phase}

The committer $C$ and the receiver $R$ receive common input $1^{n}$ and parameter $\ell$. (In [MOSV06], $\ell=\omega(\log n)$.) To commit to $v \in\{0,1\}^{n}$, the committer $C$ commits to $v$ concurrently $\ell$ times by using ExtCom as follows.

1. $C$ and $R$ execute commit stage of ExtCom $\ell$ times in parallel.

2. For each $j \in[\ell]$ in sequence, $C$ and $R$ do the following.

(a) $R$ sends the challenge message of ExtCom for the $j$-th session.

(b) $C$ sends the reply message of ExtCom for the $j$-th session.

\section{Decommit Phase}

$C$ sends $v$ to $R$ and decommits all the ExtCom commitments.

Figure 1: Concurrently extractable commitment CECom [MOSV06].

\section{Robust Concurrent Extraction.}

On the concurrently extractable commitment scheme CECom of [MOSV06], Goyal et al. [GLP $\left.{ }^{+} 15\right]$ showed a very useful lemma called the robust concurrent extraction lemma. Roughly speaking, this lemma states that even when the adversarial committer additionally participates in an external "small"-round protocol, the committed values can be extracted from the adversarial committer without rewinding the external protocol. More precisely, consider any PPT adversarial committer $\mathcal{A}$ that commits to multiple values in concurrent sessions of CECom - these sessions are denoted as the right sessions - and simultaneously participates in an execution of an arbitrary protocol $\Pi:=\langle B, A\rangle$ with a honest $B$-this session is denoted as the left session. The robust concurrent extraction lemma states that for every $\mathcal{A}$, there exists an extractor $E$ that extracts the committed values from $\mathcal{A}$ in every valid right session without rewinding the external party $B$ in the left session. The extractor $E$ fails with probability that is exponentially small in $\ell-O(k \log n)$, where $\ell$ is the parameter of CECom and $k$ is the round complexity of $\Pi$; hence, $E$ fails only with negligible probability if we set $\ell:=\omega(k \log n)$. The formal statement of the robust concurrent extraction lemma is given in Appendix A. We remark that the extractor $E$ shown by [GLP $\left.{ }^{+} 15\right]$ performs the extraction by generating the main thread and the look-ahead threads as in the rewinding strategies of [PRS02, PTV12]. 


\section{Commit Phase}

The committer $C$ and the receiver $R$ receive common inputs $1^{n}$. To commit to $v \in\{0,1\}^{n}$, the committer $C$ does the following with the receiver $R$.

commit stage.

For each $i \in[n]$, the committer $C$ chooses a pair of random $n$-bit strings $\left(a_{i}^{0}, a_{i}^{1}\right)$ such that $a_{i}^{0} \oplus a_{i}^{1}=v$. Then, for each $i \in[n]$ in parallel, $C$ commits to $a_{i}^{0}$ and $a_{i}^{1}$ by using Com $\mathrm{SB}$. For each $i \in[n]$ and $b \in\{0,1\}$, let $c_{i}^{b}$ be the commitment to $a_{i}^{b}$.

challenge stage.

$R$ sends random $n$-bit string $e=\left(e_{1}, \ldots, e_{n}\right)$ to $C$.

reply stage.

For each $i \in[n], C$ decommits $c_{i}^{e_{i}}$ to $a_{i}^{e_{i}}$.

\section{Decommit Phase}

$C$ sends $v$ to $R$ and decommits $c_{i}^{b}$ to $a_{i}^{b}$ for all $i \in[n]$ and $b \in\{0,1\}$. $R$ checks whether $a_{1}^{0} \oplus a_{1}^{1}=$ $\cdots=a_{n}^{0} \oplus a_{n}^{1}=v$.

Figure 2: Extractable commitment ExtCom [PW09], which is used as a building block in the concurrently extractable commitment scheme CECom of [MOSV06].

\section{4 (One-one) CCA-secure Commitment Schemes}

We recall the definition of (one-one) CCA security and $\kappa$-robustness of commitment schemes [CLP10, LP12, KMO14].

(One-one) CCA security. Roughly speaking, a tag-based commitment scheme $\langle C, R\rangle$ (i.e., a commitment scheme that takes an $n$-bit string — a tag — as an additional input) is $C C A$-secure if it is hiding even against adversary $\mathcal{A}$ that interacts with the following committed-value oracle: The committedvalue oracle $O$ interacts with $\mathcal{A}$ as an honest receiver in many concurrent sessions of the commit phase of $\langle C, R\rangle$ using tags chosen adaptively by $\mathcal{A}$; at the end of each session, if the commitment of this session is invalid or has multiple committed values, $O$ returns $\perp$ to $\mathcal{A}$; otherwise, $O$ returns the unique committed value to $\mathcal{A}$.

More precisely, CCA-secure commitment schemes are defined as follows. Consider the following probabilistic experiment $\operatorname{IND}_{b}(\langle C, R\rangle, \mathcal{A}, n, z)$ for each $b \in\{0,1\}$. On input $1^{n}$ and auxiliary input $z$, adversary $\mathcal{A}^{O}$ adaptively chooses a pair of challenge values $v_{0}, v_{1} \in\{0,1\}^{n}$ and an $n$-bit tag id $\in\{0,1\}^{n}$. Then, $\mathcal{A}^{O}$ interacts with the challenger and obtains a commitment to $v_{b}$ with tag id. Let $y$ be the output of $\mathcal{A}$. The output of the experiment is $\perp$ if during the experiment, $\mathcal{A}$ sends $O$ any commitment using tag id. Otherwise, the output of the experiment is $y$. Let $\operatorname{IND}_{b}(\langle C, R\rangle, \mathcal{A}, n, z)$ denote the output of experiment $\operatorname{IND}_{b}(\langle C, R\rangle, \mathcal{A}, n, z)$.

Definition 1. Let $\langle C, R\rangle$ be a tag-based commitment scheme and $O$ be the committed-value oracle of $\langle C, R\rangle$. Then, $\langle C, R\rangle$ is CCA-secure (w.r.t the committed-value oracle) if for any $\mathrm{PPT}$ adversary $\mathcal{A}$, the following are computationally indistinguishable:

- $\left\{\mathrm{IND}_{0}(\langle C, R\rangle, \mathcal{A}, n, z)\right\}_{n \in \mathbb{N}, z \in\{0,1\}^{*}}$

- $\left\{\mathrm{IND}_{1}(\langle C, R\rangle, \mathcal{A}, n, z)\right\}_{n \in \mathbb{N}, z \in\{0,1\}^{*}}$ 
If $\langle C, R\rangle$ is CCA secure only against adversaries that start a single session with $O$, we say that $\langle C, R\rangle$ is one-one CCA secure. That is, one-one CCA security is defined as follows. Let one-session committed-value oracle be an oracle that is the same as the committed-value oracle except that it interacts with the adversary only in a single session of the commit parse of $\langle C, R\rangle$. Then, one-one CCA security is defined by replacing the committed-value oracle in the definition of CCA security with the one-session committed-value oracle.

Robustness. Roughly speaking, a tag-based commitment scheme is $\kappa$-robust if for any adversary $\mathcal{A}$ and any ITM $B$, the joint output of a $\kappa$-round interaction between $\mathcal{A}^{O}$ and $B$ can be simulated without $O$ by a PPT simulator.

Definition 2. Let $\langle C, R\rangle$ be a tag-based commitment scheme and $O$ be the committed-value oracle of $\langle C, R\rangle$. For any constant $\kappa \in \mathbb{N}$, we say that $\langle C, R\rangle$ is $\kappa$-robust (w.r.t. the committed-value oracle) if there exists $a$ PPT oracle machine (called simulator) $\mathcal{S}$ such that for any PPT adversary $\mathcal{A}$ and any $\kappa$-round $\mathrm{PPT} I T M B$, the following are computationally indistinguishable:

- $\left\{\text { output }_{B, \mathcal{A} O}\left[B\left(1^{n}, x, y\right) \leftrightarrow \mathcal{A}^{O}\left(1^{n}, x, z\right)\right]\right\}_{n \in \mathbb{N}, x, y, z \in\{0,1\}^{n}}$

- $\left\{\text { output }_{B, \mathcal{S}^{\mathcal{A}}}\left[B\left(1^{n}, x, y\right) \leftrightarrow \mathcal{S}^{\mathcal{A}}\left(1^{n}, x, z\right)\right]\right\}_{n \in \mathbb{N}, x, y, z \in\{0,1\}^{n}}$

Intuitively, the $\kappa$-robustness guarantees that the security of any $\kappa$-round protocol (say, the hiding property of a $\kappa$-round commitment scheme) holds even against the adversary that interacts with $O$. In fact, it is easy to see that the following proposition holds.

Proposition 1. Let $\langle C, R\rangle$ be a $\kappa$-robust commitment scheme for a constant $\kappa \in \mathbb{N}$, and let $B$ be any $\kappa$-round РPт ITM. Then,for every two sequences $\left\{y_{n}^{1}\right\}_{n \in \mathbb{N}}$ and $\left\{y_{n}^{2}\right\}_{n \in \mathbb{N}}$ such that for every PPT adversary $\mathcal{A}^{\prime}$ it holds that

- $\left\{\text { output }_{B, \mathcal{A}^{\prime}}\left[B\left(1^{n}, x, y_{n}^{1}\right) \leftrightarrow \mathcal{A}^{\prime}\left(1^{n}, x, z\right)\right]\right\}_{n \in \mathbb{N}, x, y, z \in\{0,1\}^{n}}$ and

- $\left\{\text { output }_{B, \mathcal{A}^{\prime}}\left[B\left(1^{n}, x, y_{n}^{2}\right) \leftrightarrow \mathcal{A}^{\prime}\left(1^{n}, x, z\right)\right]\right\}_{n \in \mathbb{N}, x, y, z \in\{0,1\}^{n}}$

are computationally indistinguishable, then it also hold that for every PPT adversary $\mathcal{A}$,

- $\left\{\text { output }_{B, \mathcal{A} O}\left[B\left(1^{n}, x, y_{n}^{1}\right) \leftrightarrow \mathcal{A}^{O}\left(1^{n}, x, z\right)\right]\right\}_{n \in \mathbb{N}, x, y, z \in\{0,1\}^{n}}$ and

- $\left\{\text { output }_{B, \mathcal{A} O}\left[B\left(1^{n}, x, y_{n}^{2}\right) \leftrightarrow \mathcal{A}^{O}\left(1^{n}, x, z\right)\right]\right\}_{n \in \mathbb{N}, x, y, z \in\{0,1\}^{n}}$

are computationally indistinguishable.

Proof. From the definition of $\kappa$-robustness, there exists PPT $\mathcal{S}$ such that for each $b \in\{1,2\}$, the following are computationally indistinguishable.

- output $\left._{B, \mathcal{A}^{O}}\left[B\left(1^{n}, x, y_{n}^{b}\right) \leftrightarrow \mathcal{A}^{O}\left(1^{n}, x, z\right)\right]\right\}_{n \in \mathbb{N}, x, y, z \in\{0,1\}^{n}}$

- $\left\{\text { output }_{B, \mathcal{S}^{\mathcal{P}}}\left[B\left(1^{n}, x, y_{n}^{b}\right) \leftrightarrow \mathcal{S}^{\mathcal{A}}\left(1^{n}, x, z\right)\right]\right\}_{n \in \mathbb{N}, x, y, z \in\{0,1\}^{n}}$

Also, from the assumption of the proposition, the following are computationally indistinguishable. (Notice that $\mathcal{S}^{\mathcal{A}}$ is PPT since both $\mathcal{A}$ and $\mathcal{S}$ are PPT.)

- $\left\{\text { output }_{B, \mathcal{S}}\left[B\left(1^{n}, x, y_{n}^{1}\right) \leftrightarrow \mathcal{S}^{\mathcal{A}}\left(1^{n}, x, z\right)\right]\right\}_{n \in \mathbb{N}, x, y, z \in\{0,1\}^{n}}$

- $\left\{\text { output }_{B, \mathcal{S}}\left[B\left(1^{n}, x, y_{n}^{2}\right) \leftrightarrow \mathcal{S}^{\mathcal{A}}\left(1^{n}, x, z\right)\right]\right\}_{n \in \mathbb{N}, x, y, z \in\{0,1\}^{n}}$

The proposition follows from these two indistinguishabilities. 
The scheme we use. From a result shown in $\left[\mathrm{GLP}^{+} 15\right]$, we can obtain a constant-round $\kappa$-robust one-one CCA-secure commitment scheme from one-way functions for every constant $\kappa \in \mathbb{N}$ as follows. In [GLP $\left.{ }^{+} 15\right]$, Goyal et al. constructed a $\omega(\log n)$-round CCA-secure commitment scheme from one-way functions. This scheme has $\omega(\log n)$ rounds because CECom with parameter $\ell=\omega(\log n)$ is used as a building block. The reason why $\ell$ is set to be $\omega(\log n)$ is that in the security analysis, the committed values of CECom need to be extracted when polynomially many CECom commitments are concurrently executed. In the setting of one-one CCA security, however, the security analysis works even if the committed values of CECom are extractable only when a single CECom commitment is executed. Hence, by setting $\ell:=O(1)$, we can obtain a constant-round one-one CCA-secure commitment scheme. For completeness, we give the protocol and the proof of one-one CCA security in Appendix B.

\subsection{Witness Indistinguishable Proofs and Arguments, and Special Soundness}

Definition 3. An interactive proof (or argument) system $\langle P, V\rangle$ for an $\mathcal{N} \mathcal{P}$ language $L$ with witness relation $\boldsymbol{R}_{L}$ is said to be witness indistinguishable if for every probabilistic polynomial-time adversarial verifier $V^{*}$ and for every two sequences $\left\{w_{x}^{1}\right\}_{x \in L}$ and $\left\{w_{x}^{2}\right\}_{x \in L}$ such that $w_{x}^{1}, w_{x}^{2} \in \boldsymbol{R}_{L}(x)$, the following ensembles are computationally indistinguishable.

- $\left\{\operatorname{view}_{V^{*}}\left[P\left(x, w_{x}^{1}\right) \leftrightarrow V^{*}(x)\right]\right\}_{x \in L}$

- $\left\{\operatorname{view}_{V^{*}}\left[P\left(x, w_{x}^{2}\right) \leftrightarrow V^{*}(x)\right]\right\}_{x \in L}$

If the above ensembles are statistically indistinguishable, $\langle P, V\rangle$ is said to be statistically witness indistinguishable.

We can obtain a four-round witness-indistinguishable proof system WIPOK from one-way functions by executing Blum's Hamiltonian-cycle protocol in parallel. Recall that WIPOK consists of three stages - commit, challenge, and response - and has a useful property called special soundness: Given two accepting transcripts $\left\langle\alpha_{1}, \alpha_{1}^{\prime}, \beta_{1}, \gamma_{1}\right\rangle$ and $\left\langle\alpha_{2}, \alpha_{2}^{\prime}, \beta_{2}, \gamma_{2}\right\rangle$ of statement $x \in L$ such that $\alpha_{1}=\alpha_{2}$, $\alpha_{1}^{\prime}=\alpha_{2}^{\prime}$, and $\beta_{1} \neq \beta_{2}$, we can compute a valid witness for $x \in L$.

We can obtain a statistical witness-indistinguishable argument system sWIAOK from any statistical hiding commitment scheme by instantiating Blum's Hamiltonian-cycle protocol with the statistically hiding commitment scheme. It is easy to see that this argument system satisfies special soundness in the following sense: Let us say that two accepting transcripts $\left\langle\vec{\alpha}_{1}, \beta_{1}, \gamma_{1}\right\rangle$ and $\left\langle\vec{\alpha}_{2}, \beta_{2}, \gamma_{2}\right\rangle$ are admissible if $\vec{\alpha}_{1}=\vec{\alpha}_{2}$ and $\beta_{1} \neq \beta_{2}$; then, given admissible transcripts that are generated in polynomial time, we can compute a valid witness. In particular, given admissible transcripts $\left\langle\vec{\alpha}, \beta_{1}, \gamma_{1}\right\rangle$ and $\left\langle\vec{\alpha}, \beta_{2}, \gamma_{2}\right\rangle$, we can compute a valid witness or we can decommit a commitment given in $\vec{\alpha}$ to two different values.

\subsection{Statistical Concurrent Non-malleable Zero-knowledge Arguments}

We recall the definition of (statistical) concurrent non-malleable zero-knowledge from [BPS06, $\left.\mathrm{OOR}^{+} 14\right]$, which is closely related to the definition of simulation extractability of [PR05]. Let $\langle P, V\rangle$ be an interactive argument system for a language $L \in \mathcal{N} \mathcal{P}$ with witness relation $\mathbf{R}_{L}$. For any man-in-the-middle adversary $\mathcal{A}$, let us consider a probabilistic experiment in which $\mathcal{A}$ participates in the following left and right interactions (see Figure 3). In the left interaction, $\mathcal{A}$ interacts with a honest prover $P$ of $\langle P, V\rangle$ and verifies the validity of statements $x_{1}, \ldots, x_{m}$ using identities $\mathrm{id}_{1}, \ldots, \mathrm{id}_{m}$. In the right interaction, $\mathcal{A}$ interacts with a honest verifier $V$ of $\langle P, V\rangle$ and proves the validity of statements $\widetilde{x}_{1}, \ldots, \widetilde{x}_{m}$ using identities $\widetilde{\mathrm{id}}_{1}, \ldots, \widetilde{\mathrm{id}}_{m}$. The statements proven in the left interaction, $x_{1}, \ldots, x_{m}$, are given to $P$ 
and $\mathcal{A}$ prior to the experiment. In contrast, the statements proven in the right interaction, $\widetilde{x}_{1}, \ldots, \widetilde{x}_{m}$, and the identities used in the left and the right interactions, $i d_{1}, \ldots, \mathrm{id}_{m}$ and $\widetilde{\mathrm{id}}_{1}, \ldots, \widetilde{\mathrm{id}}_{m}$, are chosen by $\mathcal{A}$ during the experiment. Let view $\mathcal{A}_{\mathcal{A}}\left(n, x_{1}, \ldots, x_{m}, z\right)$ be a random variable representing the view of $\mathcal{A}$ in the above experiment. Then, roughly speaking, $\langle P, V\rangle$ is statistical concurrent non-malleable zero-knowledge (statistical CNMZK) if for any adversary $\mathcal{A}$, there exists a PPT machine called the simulator-extractor that can statistically simulate the view of $\mathcal{A}$ in the above experiment while extracting witnesses for the statements proven by $\mathcal{A}$ in the accepted right interactions that use different identities from the left interactions. The formal definition is given below.

Definition 4. An interactive proof $\langle P, V\rangle$ for language $L$ with witness relation $\boldsymbol{R}_{L}$ is said to be statistical concurrent non-malleable zero-knowledge if for every polynomial $m(\cdot)$ and every probabilistic polynomial-time man-in-the-middle adversary $\mathcal{A}$ that participates in at most $m=m(n)$ concurrent executions, there exists a probabilistic polynomial-time machine $\mathcal{S} \mathcal{E}$ called simulator-extractor such that the following hold.

1. Let sim-view $\left(n, x_{1}, \ldots, x_{m}, z\right)$ be a random variable representing the first output of $\mathcal{S} \mathcal{E}\left(n, x_{1}, \ldots\right.$, $\left.x_{m}, z\right)$. Then, the following ensembles are statistically indistinguishable.

- $\left\{\operatorname{view}_{\mathcal{A}}\left(n, x_{1}, \ldots, x_{m}, z\right)\right\}_{n \in \mathbb{N}, x_{1}, \ldots, x_{m} \in L \cap\{0,1\}^{n}, z \in\{0,1\}^{*}}$

- $\left\{\operatorname{sim}-\operatorname{view}\left(n, x_{1}, \ldots, x_{m}, z\right)\right\}_{n \in \mathbb{N}, x_{1}, \ldots, x_{m} \in L \cap\{0,1\}^{n}, z \in\{0,1\}^{*}}$

2. For every $n \in \mathbb{N}, x_{1}, \ldots, x_{m} \in L \cap\{0,1\}^{n}$, and $z \in\{0,1\}^{*}$, the following holds. Let (view, $\left\{\widetilde{w}_{i}\right\}_{i \in[m]}$ ) denote the output of $\mathcal{S} \mathcal{E}\left(n, x_{1}, \ldots, x_{m}, z\right)$. Let $\widetilde{x}_{1}, \ldots, \widetilde{x}_{m}$ be the statements of the right interaction in view, and let $\mathrm{id}_{1}, \ldots, \mathrm{id}_{m}$ and $\widetilde{\mathrm{id}}_{1}, \ldots, \widetilde{\mathrm{id}}_{m}$ be the identities of the left and the right interactions in view, respectively. Then, except with negligible probability, we have $\left(\widetilde{x}_{i}, \widetilde{w}_{i}\right) \in \boldsymbol{R}_{L}$ for every $i \in[\mathrm{m}]$ such that the $i$-th right interaction is accepting and $\tilde{\mathrm{id}}_{i} \neq \mathrm{id}_{j}$ holds for every $j \in[m]$.

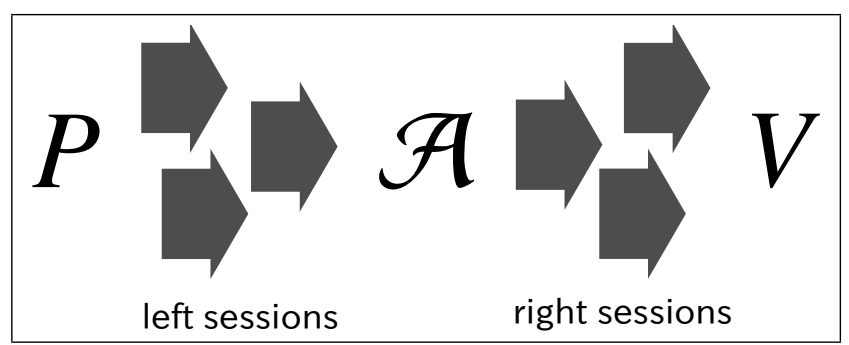

Figure 3: Left sessions and right sessions.

\section{Our Statistical Concurrent Non-malleable ZK Argument}

We show that a statistical concurrent non-malleable zero-knowledge argument can be constructed from any statistically hiding commitment scheme.

Theorem 2. Assume the existence of statistically hiding commitment schemes with round complexity $R_{\mathrm{SH}}(n)$. Then, there exists an $\omega\left(R_{\mathrm{SH}}(n) \log n\right)$-round statistical concurrent non-malleable zeroknowledge argument SCNMZK.

Since poly $(n)$-round statistically hiding commitment schemes can be constructed from one-way functions $\left[\mathrm{HNO}^{+} 09\right]$ and constant-round ones can be constructed from a family of collision-resistant hash functions [NY89, DPP98], our main theorem (Theorem 1) follows from Theorem 2. 
Proof of Theorem 2. In SCNMZK, we use the following building blocks.

- Two-round statistically binding commitment scheme Com $\mathrm{SB}$.

- Constant-round 4-robust one-one CCA-secure commitment scheme CCACom ${ }^{1: 1}$.

- Four-round witness-indistinguishable proof of knowledge WIPOK, which is a parallel version of Blum's Hamiltonian-cycle protocol.

- $\left(R_{\mathrm{SH}}(n)+2\right)$-round statistical witness-indistinguishable argument of knowledge sWIAOK, which is a parallel version of Blum's Hamiltonian-cycle protocol that is instantiated with a $R_{\mathrm{SH}}(n)$ round statistically hiding commitment scheme $\mathrm{Com}_{\mathrm{SH}}$.

- $\omega\left(R_{\mathrm{SH}}(n) \log n\right)$-round concurrently extractable commitment scheme CECom, which is the scheme of [MOSV06] with parameter $\ell=\omega\left(R_{\mathrm{SH}}(n) \log n\right)$. From the robust concurrent extraction lemma [GLP $\left.{ }^{+} 15\right]$, we can extract the committed values from any adversarial committer even when it additionally participates in any $O\left(R_{\mathrm{SH}}(n)\right)$-round external protocol.

As explained in Section 3, all of the above building blocks can be constructed from $R_{\mathrm{SH}}(n)$-round statistically hiding commitment schemes (or from one-way functions, which can be obtained from statistically hiding commitment schemes).

Protocol sCNMZK is shown in Figure 4. We prove its soundness in Section 4.1 and prove its statistical CNMZK property in Section 4.2.

\subsection{Proof of Soundness}

Lemma 1. Protocol sCNMZK is sound.

Proof. Assume for contradiction that there exists an adversarial prover $P^{*}$ that breaks the soundness of SCNMZK. It follows from the argument-of-knowledge property of sWIAOK that we can extract $r_{V}$ from $P^{*}$ in Stage III with non-negligible probability, where $r_{V}$ is the value committed to by the verifier in Stage I-1. In the following, we consider a sequence of hybrid experiments in which the verifier is gradually modified so that $P^{*}$ receives no information about $r_{V}$ in the last hybrid, and then we derive a contradiction by showing that $r_{V}$ is still extractable with non-negligible probability in the last hybrid.

Hybrid $H_{0}$ is an experiment in which a honest verifier interacts with $P^{*}$ and then a witness is extracted in Stage III by the knowledge extractor of sWIAOK. The output of $H_{0}$ is the witness extracted in Stage III. From the above observation, the output of $H_{0}$ is $r_{V}$ with non-negligible probability.

Hybrid $H_{1}$ is the same as $H_{0}$ except that (i) the committed value $r_{P}$ of the CCACom ${ }^{1: 1}$ commitment in Stage II-1 is extracted by the one-session committed-value oracle $O$ of CCACom ${ }^{1: 1}$ and (ii) the committed value of the CECom commitment in Stage II-2 is switched from $0^{n}$ to $r_{P}$.

Note that, basically, the only difference between $H_{0}$ and $H_{1}$ is the value committed to with CECom in Stage II-2. However, since the execution of $H_{1}$ involves a super-polynomial-time computation (i.e., the extraction of $r_{P}$ ), we cannot directly use the hiding property of CECom to argue that the output of $H_{1}$ is indistinguishable from that of $H_{0}$. Nevertheless, since $H_{1}$ can be executed in polynomial-time given access to the one-session committed-value oracle of CCACom $^{1: 1}$, we can show the indistinguishability between the output of $H_{1}$ and that of $H_{0}$ by combining the hiding property of CECom with the robustness of CCACom ${ }^{1: 1}$ (cf. Proposition 1 in Section 3.4). 
Input. The common input is statement $x \in L$ and identity id $\in\{0,1\}^{n}$. The prover's private input is witness $w \in \mathbf{R}_{L}(x)$.

\section{Stage I. ( $V$ commits to trapdoor)}

1. $V$ chooses random $r_{V} \in\{0,1\}^{n}$ and commits to $r_{V}$ by using ComsB. Let $\left(r_{V}, d\right)$ be the decommitment of this commitment.

2. $V$ commits to $\left(r_{V}, d\right)$ by using CECom.

Stage II. ( $V$ proves knowledge of trapdoor)

1. $P$ chooses random $r_{P} \in\{0,1\}^{n}$ and commits to $r_{P}$ by using CCACom ${ }^{1: 1}$ with tag id.

2. $V$ commits to $0^{n}$ by using CECom.

3. $P$ decommits the CCACom ${ }^{1: 1}$ commitment in Stage II- 1 to $r_{P}$.

4. $V$ proves the following by using WIPOK:

- the committed value of the CECom commitment in Stage I-2 is a valid decommitment of the ComsB commitment in Stage I-1, or

- the committed value of the CECom commitment in Stage II-2 is $r_{P}$.

Stage III. ( $P$ proves $x \in L$ or knowledge of trapdoor)

1. $P$ proves the following by using sWIAOK:

- $x \in L$, or

- there exists $\left(r_{V}^{\prime}, d^{\prime}\right)$ such that $\left(r_{V}^{\prime}, d^{\prime}\right)$ is a valid decommitment of the Com commitment in Stage I-1.

Figure 4: Statistical concurrent non-malleable zero-knowledge argument sCNMZK. 
CoMmENT: Formally, since CCACom ${ }^{1: 1}$ is robust only w.r.t. 4-round protocols, we need to consider a sequence of intermediate hybrids in which the CECom commitment is gradually modified by switching the committed values of the ExtCom commitments one by one in CECom. Since ExtCom has only four rounds, the 4-robustness of $\mathrm{CCACom}^{1: 1}$ guarantees that the outputs of these intermediate hybrids are indistinguishable.

Hybrid $H_{2}$ is the same as $H_{1}$ except that the WIPOK proof in Stage II-4 is computed by using a witness for the fact that the committed value of the CECom commitment in Stage II-2 is $r_{P}$.

Similar to the above, the indistinguishability between the output of $H_{2}$ and that of $H_{1}$ follows from the witness-indistinguishability of WIPOK and the robustness of CCACom ${ }^{1: 1}$.

Hybrid $H_{3}$ is the same as $H_{2}$ except that in Stage I-2, the committed value of the CECom commitment is switched from $\left(r_{V}, d\right)$ to $\left(0^{\left|r_{V}\right|}, 0^{|d|}\right)$.

The indistinguishability between the output of $\mathrm{H}_{3}$ and $\mathrm{H}_{2}$ follows from the hiding property of CECom (or, more precisely, the hiding property of ExtCom used in CECom) and the robustness of CCACom ${ }^{1: 1}$.

Hybrid $H_{4}$ is the same as $H_{3}$ except that in Stage I-1, the committed value of the Com $\mathrm{SB}$ commitment is switched from $r_{V}$ to $0^{n}$.

The indistinguishability between the output of $H_{4}$ and that of $H_{3}$ follows from the hiding property of $\mathrm{Com}_{\mathrm{SB}}$ and the robustness of CCACom ${ }^{1: 1}$.

From the above, the probability that the output of $H_{4}$ is $r_{V}$ is non-negligible. However, since $P^{*}$ receives no information about $r_{V}$ in $H_{4}$, this probability must be negligible. Thus, we reach a contradiction.

\subsection{Proof of Statistical CNMZK Property}

\section{Simulator-extractor $\mathcal{S} \mathcal{E}$.}

Recall that to prove the statistical CNMZK property, we need to show a simulator-extractor that statistically simulates the view of the adversary $\mathcal{A}$ while extracting a witness in every accepted right session. We construct our simulator-extractor step by step. First, we construct a super-polynomialtime simulator $\hat{\mathcal{S}}$ that simulates the view of $\mathcal{A}$ but does not extract witnesses in the right seasons. Next, we construct a super-polynomial-time simulator-extractor $\hat{\mathcal{S} E}$ that simulates the view of $\mathcal{A}$ by executing $\hat{\mathcal{S}}$ and then extracts the witnesses by rewinding $\hat{\mathcal{S}}$. Finally, we construct a polynomial-time simulator-extractor $\mathcal{S} \mathcal{E}$ that emulates the execution of $\hat{\mathcal{S} \mathcal{E}}$ in polynomial time.

Remark 1 . In the following, we use the hat symbol in the names of simulators and simulator-extractors if they run in super-polynomial time (e.g., $\hat{\mathcal{S}}$ and $\hat{\mathcal{S} E}$ ).

Remark 2. In the following, we use the tilde symbol in the names of the messages of sCNMZK if they are the messages of the right sessions (e.g., $\widetilde{r}_{V}$ and $\widetilde{r}_{P}$ ). If necessary, we use subscript to denote the index of the session.

Super-polynomial-time simulator $\hat{\mathcal{S}}$. First, the simulator $\hat{\mathcal{S}}$ simulates the view of $\mathcal{A}$ in superpolynomial time as follows. $\hat{\mathcal{S}}$ internally invokes $\mathcal{A}$ and interacts with $\mathcal{A}$ as provers and verifiers in the following way.

- In each left session, $\hat{\mathcal{S}}$ interacts with $\mathcal{A}$ in the same way as a honest prover except for the following. In Stage I-2, $\hat{\mathcal{S}}$ extracts the committed value $\left(r_{V}, d\right)$ of the CECom commitment by 
brute force. (If the committed value is not uniquely determined, $\left(r_{V}, d\right)$ is defined to be $(\perp, \perp)$.) In Stage III, $\hat{\mathcal{S}}$ checks whether $\left(r_{V}, d\right)$ is a valid decommitment of the Com $\mathrm{SB}_{\mathrm{B}}$ commitment in Stage I- 1 ; if so, $\hat{\mathcal{S}}$ gives a sWIAOK proof by using $\left(r_{V}, d\right)$ as a witness; otherwise, $\hat{\mathcal{S}}$ terminates with output fail.

- In each right session, $\hat{\mathcal{S}}$ interacts with $\mathcal{A}$ in the same way as a honest verifier.

Finally, $\hat{\mathcal{S}}$ outputs the view of internal $\mathcal{A}$. Notice that $\hat{\mathcal{S}}$ does not rewind $\mathcal{A}$.

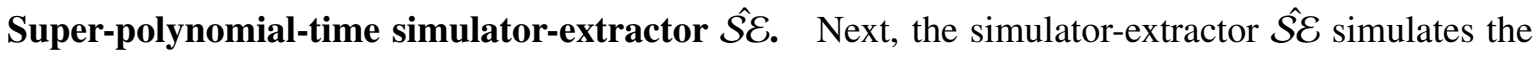
view of $\mathcal{A}$ in super-polynomial time and extracts witnesses in the accepted right sessions as follows. First, $\hat{\mathcal{S} E}$ simulates the view of $\mathcal{A}$ by executing $\hat{\mathcal{S}}$. We call this execution of $\hat{\mathcal{S}}$ the wI-main thread. Next, for each $i \in[m]$, if the $i$-th right session is accepted on the wI-main thread and uses a different

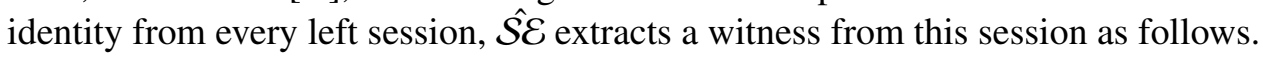

- $\hat{\mathcal{S E}}$ rewinds the wI-main thread until the point just before the challenge message of sWIAOK of

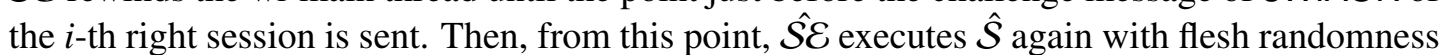

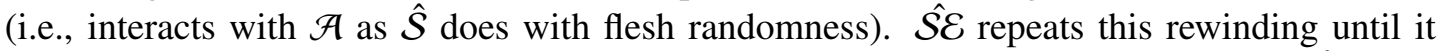
obtains another accepting transcript of the $i$-th right session. We call each execution of $\hat{\mathcal{S}}$ in this step a wI-auxiliary thread.

- After obtaining two accepting transcripts of the $i$-th right session (one is on the wI-main thread and the other is on an wI-auxiliary thread), $\hat{\mathcal{S E}}$ extracts a witness from sWIAOK by using the

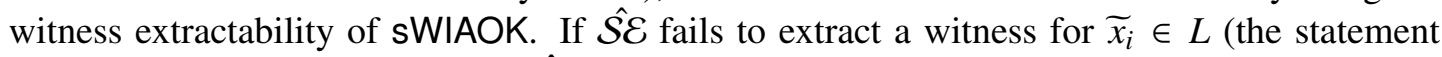

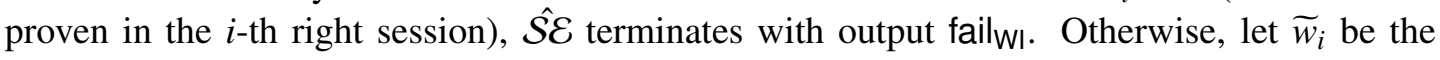
extracted witness.

If the $i$-th right session is not accepted or uses the same identity as a left session, define $\widetilde{w}_{i} \stackrel{\text { def }}{=} \perp$. The output of $\hat{\mathcal{S} \mathcal{E}}$ is (view, $\left\{\widetilde{w}_{i}\right\}_{i \in[m]}$ ), where view is the view of $\mathcal{A}$ on the wI-main thread.

Polynomial-time simulator-extractor $\mathcal{S} \mathcal{E}$. Finally, the simulator-extractor $\mathcal{S} \mathcal{E}$ emulates the execution of $\hat{\mathcal{S}}$ in polynomial time as follows. First, $\mathcal{S} \mathcal{E}$ emulates the wI-main thread in polynomial time as follows.

- $\mathcal{S} \mathcal{E}$ internally invokes $\mathcal{A}$ and interacts with $\mathcal{A}$ as $\hat{\mathcal{S}}$ does except that in each left session, $\mathcal{S} \mathcal{E}$ extracts $\left(r_{V}, d\right)$ by using the concurrent extractability of CECom instead of by brute force. Recall that a concurrent extraction of CECom involves the generation of a main thread and many look-ahead threads. We call the main thread generated during the concurrent extraction of CECom the CEC-main thread, and call the look-ahead threads generated during the concurrent extraction of CECom the cEC-auxiliary threads. ${ }^{6}$ (See Figure 5.)

Next, for each $i \in[m]$, if the $i$-th right session is accepted on the emulated wI-main thread and uses a different identity from every left session, $\mathcal{S} \mathcal{E}$ emulates wI-auxiliary threads as follows.

- $\mathcal{S E}$ rewinds the emulation of the wI-main thread until the point just before the challenge message of SWIAOK of the $i$-th right session is sent on the CEC-main thread. Then, from this point, $\hat{\mathcal{S E}}$ emulates the wI-main thread again with flesh randomness (i.e., generates the rest of CECmain thread and cEc-auxiliary threads with flesh randomness). $\mathcal{S} \mathcal{E}$ repeats this rewinding until it obtains another accepted transcript of the $i$-th right session on an emulated wI-auxiliary thread.

Let (view, $\left\{\widetilde{w}_{i}\right\}_{i \in[m]}$ ) be the output of the emulated $\hat{\mathcal{S E}}$. Then, $\mathcal{S} \mathcal{E}$ outputs (view, $\left\{\widetilde{w}_{i}\right\}_{i \in[m]}$ ).

\footnotetext{
${ }^{6}$ Note that the wI-main thread is also a cEC-main thread.
} 


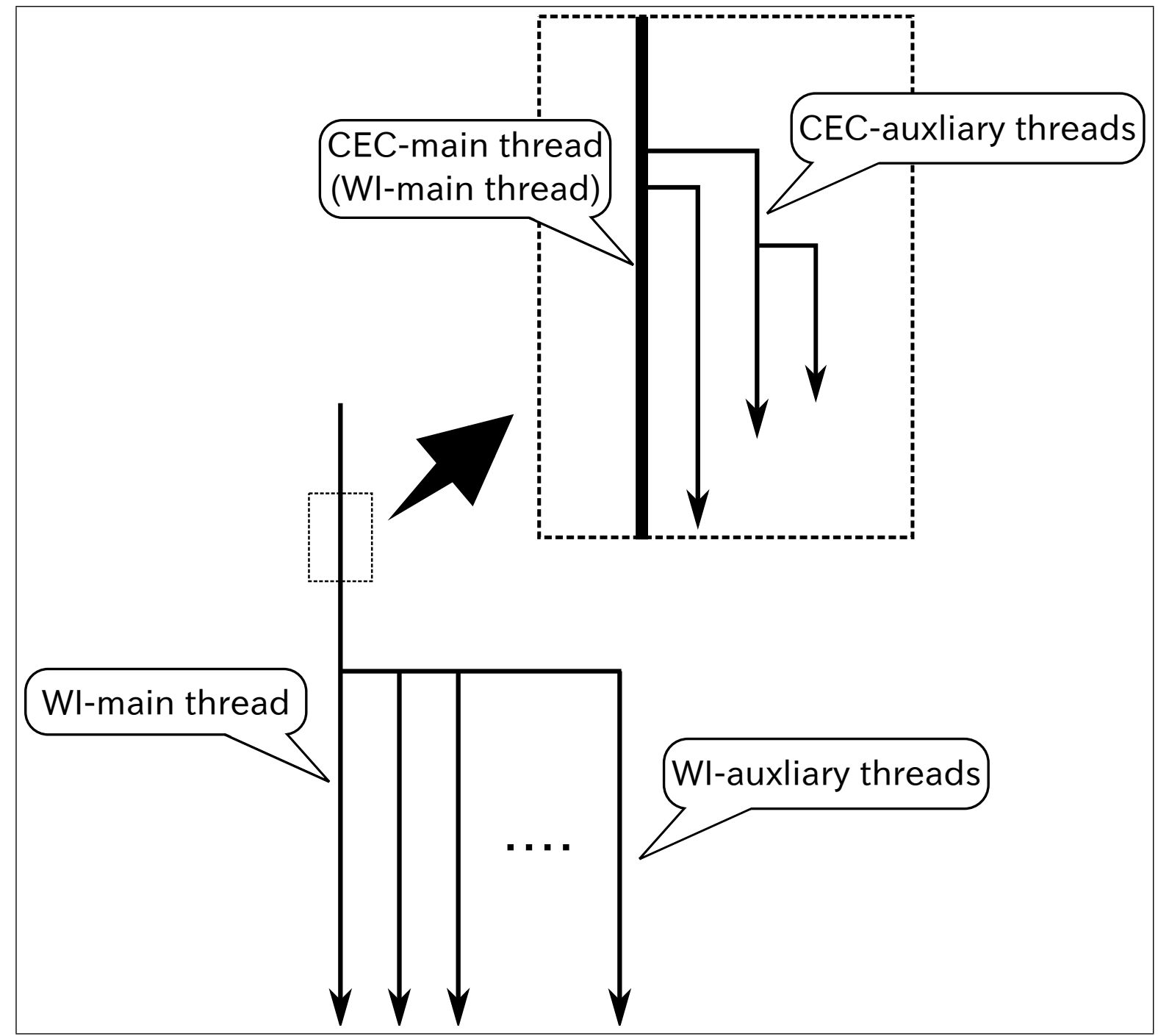

Figure 5: wI-main thread, wI-auxiliary thread, cec-main thread, and CEC-auxiliary thread. 


\section{Analysis of poly-time simulator-extractor $\mathcal{S} \mathcal{E}$.}

To prove the statistical CNMZK property, we show that $\mathcal{S} \mathcal{E}$ statistically simulates the view of $\mathcal{A}$ and also extracts witnesses for the statements in the right sessions.

Lemma 2. The view of $\mathcal{A}$ simulated by $\mathcal{S E}$ is statistically indistinguishable from the view of $\mathcal{A}$ in the real experiment. Furthermore, except with negligible probability, $\mathcal{S} \mathcal{E}$ outputs witnesses for the statements proven by $\mathcal{A}$ in the accepted right sessions that use different identities from the left sessions.

Proof. In this proof, we use the following claim, which states that the super-polynomial-time simulatorextractor $\hat{\mathcal{S E}}$ statistically simulates the view of $\mathcal{A}$ and also extracts the witnesses from the right sessions.

Claim 1. The view of $\mathcal{A}$ simulated by $\hat{\mathcal{S E}}$ is statistically indistinguishable from the view of $\mathcal{A}$ in the real experiment. Furthermore, except with negligible probability, $\hat{\mathcal{S E}}$ outputs witnesses for the statements proven by $\mathcal{A}$ in the accepted right sessions that use different identities from the left sessions.

Before proving this claim, we finish the proof of Lemma 2. Given Claim 1, we can prove Lemma 2 by showing that the output of $\mathcal{S} \mathcal{E}$ is statistically indistinguishable from that of $\hat{\mathcal{S}}$. Roughly speaking, this indistinguishability can be shown by observing the following.

- In $\mathcal{S E}$, the emulation of $\hat{\mathcal{S E}}$ is perfect if in every left session that reaches Stage III, the value extracted by the concurrent extractability of CECom is equal to the value that would be extracted by brute force.

- In every such left session, the value extracted by the concurrent extractability of CECom is indeed equal to the value that would be extracted by brute force except with negligible probability. This is because the CECom commitment in Stage I-2 is valid in every such left session except with negligible probability, which in turn is because of the soundness of WIPOK in Stage II-4 and the hiding property of CCACom ${ }^{1: 1}$ in Stage II-1.

We note that there is a subtlety since the concurrent extraction of CECom itself is rewound in $\mathcal{S E}$ when the witnesses are extracted from the right sessions. A formal argument is given below.

Let CEC-BAD be the event that during the execution of $\mathcal{S} \mathcal{E}$, in a left session that reaches Stage III, the value extracted from the CECom commitment in Stage I-2 is different from the value that would be extracted by the brute-force extraction. Let $\epsilon$ be the probability that CEC-BAD occurs during the emulation of the wI-main thread (i.e., during the emulation of the execution of $\hat{\mathcal{S}}$ ). From the concurrent extractability of CECom, if the CECom commitment in Stage I-2 is valid except with negligible probability, CEC-BAD occurs only with negligible probability. Hence, we obtain $\epsilon=\operatorname{negl}(n)$ from the following claim.

Claim 2. In $\hat{\mathcal{S}}$, the following holds except with negligible probability: In every left session that reaches Stage III, the CECom commitment in Stage I-2 of this session is valid and its committed value is a valid decommitment of the $\mathrm{Com}_{\mathrm{SB}}$ commitment in Stage I-1.

The proof of Claim 2 is given after this proof.

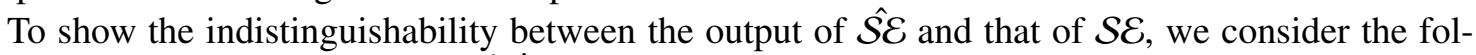
lowing hybrid simulator-extractor $\hat{\mathcal{S}} \mathcal{E}^{\prime}$ and $\mathcal{S} \mathcal{E}^{\prime}$.

- $\hat{\mathcal{S} \mathcal{E}^{\prime}}$ (resp., $\mathcal{S} \mathcal{E}^{\prime}$ ) is the same as $\hat{\mathcal{S E}}$ (resp., $\mathcal{S} \mathcal{E}$ ) except that for each $i \in[m]$, it terminates with output time-out if it does not obtain another accepting transcript of the $i$-th right session after rewinding the wI-main thread (resp., the emulation of the wI-main thread) $1 / \epsilon^{1 / 4}$ times. 
First, we show that the output of $\hat{\mathcal{S E}}$ and that of $\hat{\mathcal{S} \mathcal{E}^{\prime}}$ are statistically indistinguishable. From the definition of $\hat{\mathcal{S} \mathcal{E}^{\prime}}$, this indistinguishability holds if $\hat{\mathcal{S}}^{\prime}$ outputs time-out with at most negligible probability. Thus, to show this indistinguishability, it suffices to show that the probability that $\hat{\mathcal{S E}}$ rewinds wI-main thread more than $1 / \epsilon^{1 / 4}$ times during the witness extraction of a right session is negligible. To show that this probability is negligible, we do the following. For each $i \in[m]$, let $T_{i}$ be the random variable representing the number of rewinding during the witness extraction of the $i$-th right session in $\hat{\mathcal{S E}}$. From a standard " $p \times 1 / p$ " argument, we can show that we have $\mathrm{E}\left[T_{i}\right]=1$ for every $i \in[m] .{ }^{7}$ Thus, from Marcov's inequality, we have

$$
\operatorname{Pr}\left[T_{i}>1 / \epsilon^{1 / 4}\right] \leq \epsilon^{1 / 4}=\operatorname{negl}(n)
$$

for every $i \in[m]$. Thus, from union bound, we have

$$
\operatorname{Pr}\left[\exists i \in[m] \text { s.t. } T_{i}>1 / \epsilon^{1 / 4}\right] \leq m \cdot \operatorname{negl}(n)=\operatorname{negl}(n) .
$$

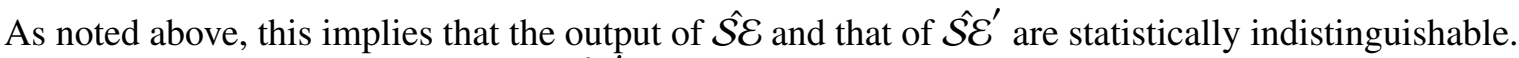

Next, we show that the output of $\hat{\mathcal{E}}^{\prime}$ and that of $\mathcal{S} \mathcal{E}^{\prime}$ are statistically indistinguishable. Since the only difference between $\hat{\mathcal{S} \mathcal{E}^{\prime}}$ and $\mathcal{S} \mathcal{E}^{\prime}$ is how the committed values are extracted from CECom, this indistinguishability holds if CEC-BAD occurs in $\mathcal{S} \mathcal{E}^{\prime}$ with at most negligible probability. For $\ell \in \mathbb{N}$, let $S T_{\ell}$ be the random variable representing the internal state of $S \mathcal{E}^{\prime}$ at the time that $\mathcal{A}$ has sent the $\ell$-th messages on the CEC-main thread during the emulation of wI-main thread. Let CEC-BAD main be the event that CEC-BAD occurs during the emulation of the wI-main thread. We say that an internal state st of $\mathcal{S} \mathcal{E}^{\prime}$ is good w.r.t. $\ell$ if we have $\operatorname{Pr}\left[\right.$ CEC-BAD main $\left.\mid S T_{\ell}=\mathrm{st}\right] \leq \epsilon^{1 / 2}$. Let $\mathrm{GOOD}_{\ell}$ be the event that $S T_{\ell}=$ st holds for an internal state st that is good w.r.t. $\ell$. Then, for any $\ell$, we have

$$
\operatorname{Pr}[\text { CEC-BAD main }] \geq \operatorname{Pr}\left[\text { CEC-BAD main } \mid \neg G \mathrm{OOD}_{\ell}\right] \operatorname{Pr}\left[\neg \mathrm{GOOD}_{\ell}\right] \geq \epsilon^{1 / 2} \cdot \operatorname{Pr}\left[\neg \mathrm{GOOD}_{\ell}\right] .
$$

Then, since we have $\operatorname{Pr}\left[\mathrm{CEC}-\mathrm{BAD}_{\text {main }}\right]=\epsilon$ from the definition of $\epsilon$, we have

$$
\operatorname{Pr}\left[\neg \mathrm{GOOD}_{\ell}\right] \leq \epsilon^{1 / 2}=\operatorname{negl}(n)
$$

for every $\ell$. Thus, from union bound, we have

$$
\operatorname{Pr}\left[\bigvee_{\ell} \neg \mathrm{GOOD}_{\ell}\right] \leq \operatorname{negl}(n) .
$$

Let GOOD be the event that $\mathrm{GOOD}_{\ell}$ occurs for every $\ell$. Then, from Equation (1), we have $\operatorname{Pr}[\mathrm{GOOD}] \geq$ $1-\operatorname{negl}(n)$. For $i \in[m]$, let CEC-BAD $i$ be the event that CEC-BAD occurs during the witness extraction of the $i$-th right session. Then, since the emulation of each wI-auxiliary thread proceeds identically with that of the wI-main thread, and since for each $i \in[m]$ there are at most $1 / \epsilon^{1 / 4}$ wI-auxiliary threads during the witness extraction of the $i$-th right session, we have

$$
\operatorname{Pr}\left[\mathrm{CEC}-\mathrm{BAD}_{i} \mid \mathrm{GOOD}\right] \leq \frac{1}{\epsilon^{1 / 4}} \cdot \epsilon^{1 / 2}=\epsilon^{1 / 4} .
$$

\footnotetext{
${ }^{7}$ For any prefix $\rho$ of the transcript immediately before the challenge message of sWIAOK of the $i$-th right session, let $p$ be the probability that the $i$-th right session is accepted when the prefix of the transcript is $\rho$. Then, we have $\mathrm{E}\left[T_{i} \mid \operatorname{prefix}_{\rho}\right]=p \cdot 1 / p=1$, where $\operatorname{prefix}_{\rho}$ is the event that the prefix of the transcript is $\rho$. Thus, we have $\mathrm{E}\left[T_{i}\right]=\sum_{\rho} \mathrm{E}\left[T_{i} \mid \operatorname{prefix}_{\rho}\right] \operatorname{Pr}\left[\operatorname{prefix}_{\rho}\right]=1$.
} 
Thus, we have

$$
\begin{aligned}
\operatorname{Pr}[\text { CEC-BAD }] & =\operatorname{Pr}\left[\text { CEC-BAD }{ }_{\text {main }}\right]+\sum_{i=1}^{m} \operatorname{Pr}[\text { CEC-BAD } i] \\
& \leq \operatorname{Pr}\left[{\text { CEC }-B \text { AD }}_{\text {main }}\right]+\sum_{i=1}^{m}(\operatorname{Pr}[\neg G O O D]+\operatorname{Pr}[\text { CEC-BAD } i \mid \text { GOOD }]) \\
& \leq \epsilon+\sum_{i=1}^{m}\left(\operatorname{negl}(n)+\epsilon^{1 / 4}\right) \\
& =\operatorname{negl}(n)
\end{aligned}
$$

As noted above, this implies that the output of $\hat{\mathcal{S} \mathcal{E}^{\prime}}$ and that of $\mathcal{S} \mathcal{E}^{\prime}$ are statistically indistinguishable.

Finally, we show that the output of $\mathcal{S} \mathcal{E}^{\prime}$ and that of $\mathcal{S} \mathcal{E}$ are statistically indistinguishable. Since the output of $\hat{\mathcal{S}} \mathcal{E}^{\prime}$ and that of $\mathcal{S} \mathcal{E}^{\prime}$ are statistically indistinguishable and since $\hat{\mathcal{S} \mathcal{E}^{\prime}}$ outputs time-out with at most negligible probability, $\mathcal{S} \mathcal{E}^{\prime}$ outputs time-out with at most negligible probability. Then, since $\mathcal{S} \mathcal{E}^{\prime}$ is identical to $\mathcal{S} \mathcal{E}$ unless $\mathcal{S} \mathcal{E}^{\prime}$ outputs time-out, the output of $\mathcal{S} \mathcal{E}^{\prime}$ and that of $\mathcal{S} \mathcal{E}$ are statistically indistinguishable.

Proof of Claim 2. Recall that Claim 2 states that during the execution of $\hat{\mathcal{S}}$, in every left session that reaches Stage III, the CECom commitment in Stage I-2 is valid and its committed value is a valid decommitment of the Com $\mathrm{SB}_{\mathrm{B}}$ commitment in Stage I-1.

Let us say that a left session is bad if it reaches Stage III and either the CECom commitment in Stage I-2 is invalid or its committed value is not a valid decommitment of the Com $\mathrm{SB}_{\mathrm{B}}$ commitment in Stage I-1; a left session is good if it is not bad. What we need to prove is that every left session is good except with negligible probability.

Roughly speaking, the proof proceeds as follows. From the soundness of WIPOK, if a left session is bad, then in Stage II-2 of this left session, the committed value of the CECom commitment is $r_{P}$, which is the committed value of the CCACom ${ }^{1: 1}$ commitment in Stage II- 1 ; thus, before $r_{P}$ is decommitted to in Stage II-3, we can obtain $r_{P}$ by extracting the committed value from CECom in Stage II-2. This itself does not contradict the hiding property of CCACom ${ }^{1: 1}$ since $\hat{\mathcal{S}}$ runs in super-polynomial time in the brute-force extraction of CECom. Thus, we consider a hybrid simulator in which the brute-force extraction of CECom is replaced with the concurrent extraction of CECom. Here, since we want to use the hiding property of $\operatorname{CCACom}^{1: 1}$, we use the robust concurrent extraction of CECom so that the CCACom ${ }^{1: 1}$ commitment in a left session is not rewound. For details, see below.

Assume for contradiction that there exists $i \in[m]$ such that the $i$-th left session is bad with nonnegligible probability. (Here, the indices of the left sessions are defined by the order in which Stage II-3 begins; the reason why we define the indices in this way will become clear later.) Then, there exists $i^{*} \in[m]$ such that the first $\left(i^{*}-1\right)$ left sessions are good except with negligible probability but the $i^{*}$-th left session is bad with non-negligible probability. Note that from the soundness of WIPOK, when the $i^{*}$-th left session is bad, then the CECom commitment in Stage II-2 of the $i^{*}$-th left session is valid and its committed value is $r_{P}$ except with negligible probability, where $r_{P}$ is the value committed to in Stage II-1 of the $i^{*}$-th left session. In the following, we use BAD to denote the event that the $i^{*}$-th left session is bad, and use CHEAT to denote the event that the committed value of the CECom commitment in Stage II-2 is $r_{P}$ in the $i^{*}$-th left session. Then, let us consider the following hybrids.

Hybrid $\hat{\mathcal{S}}_{0}$ is the same as $\hat{\mathcal{S}}$. From our assumption, BAD occurs in $\hat{\mathcal{S}}_{0}$ with non-negligible probability. Thus, from the above argument, CHEAT occurs in $\hat{\mathcal{S}}_{0}$ with non-negligible probability. 
Hybrid $\hat{\mathcal{S}}_{1}$ is the same as $\hat{\mathcal{S}}_{0}$ except that $\hat{\mathcal{S}}_{1}$ terminates just before Stage II- 3 of the $i^{*}$-th left session begins. Clearly, CHEAT still occurs in $\hat{\mathcal{S}}_{1}$ with non-negligible probability.

Hybrid $\mathcal{S}_{1}$ emulates $\hat{\mathcal{S}}_{1}$ in polynomial time as follows.

- At the beginning, a random left session $s$ is chosen. (Here, we guess that session $s$ is the $i^{*}$-th left session.)

- In every left session, in Stage I-2, the committed value $\left(r_{V}, d\right)$ is extracted by the robust concurrent extractor of CECom in such a way that the CCACom ${ }^{1: 1}$ commitment of session $s$ is not rewound. In addition, in the left session $s$, the committed value is also extracted from the CECom commitment in Stage II-2.

Note that in every left session in which Stage III is executed, the CECom commitment in Stage I-2 is valid except with negligible probability (since such a session is one of the first $\left(i^{*}-1\right)$ left sessions and therefore it is good except with negligible probability). Thus, the values extracted from the concurrent extractor are equal to the values that would be extracted by the brute-force extraction except with negligible probability; therefore, $\mathcal{S}_{1}$ statistically emulates $\hat{\mathcal{S}}_{1}$, and CHEAT occurs in $\mathcal{S}_{1}$ with non-negligible probability.

Note that session $s$ is the $i^{*}$-th left session with non-negligible probability. Then, since CHEAT occurs in $\mathcal{S}_{1}$ with non-negligible probability, the value extracted from the CECom commitment in Stage II-2 of session $s$ is $r_{P}$ with non-negligible probability, where $r_{P}$ is the value committed to in Stage II-1 of session $s$. Then, since the CCACom ${ }^{1: 1}$ commitment in Stage II-1 of session $s$ is not rewound in $\mathcal{S}_{1}$, we can break the hiding property of CCACom ${ }^{1: 1}$. Thus, we reach a contradiction.

\section{Analysis of super-poly-time simulator-extractor $\hat{\mathcal{S}} \mathcal{E}$.}

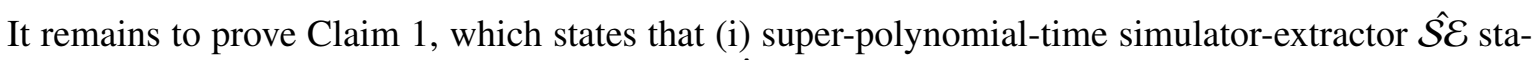
tistically simulates the real view of $\mathcal{A}$ and (ii) $\hat{\mathcal{S} \mathcal{E}}$ also extracts a valid witness from every accepted right session in the simulated view.

Proof of Claim 1. First, we observe that the output of $\hat{\mathcal{S}}$ is statistically indistinguishable from the

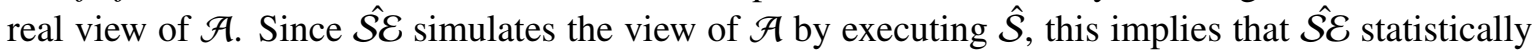
simulates the real view of $\mathcal{A}$. Recall that in $\hat{\mathcal{S}}$, each left session is simulated by extracting $\left(r_{V}, d\right)$ from the CECom commitment in Stage I-2 and giving a sWIAOK proof in Stage III with witness $\left(r_{V}, d\right)$. From Claim 2 (which states that the CECom commitment in Stage I-2 is a valid commitment to a valid decommitment of the Com $\mathrm{SB}_{\mathrm{B}}$ commitment in Stage I-1 in every session that reaches Stage III), the value $\left(r_{V}, d\right)$ that is extracted from the CECom commitment in Stage I-2 is a valid decommitment

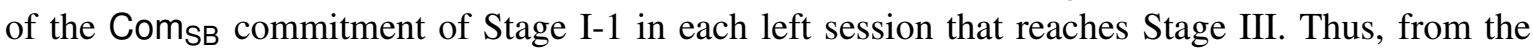
statistical witness indistinguishability of sWIAOK, the output of $\hat{\mathcal{S}}$ is statistically indistinguishable from the real view of $\mathcal{A}$.

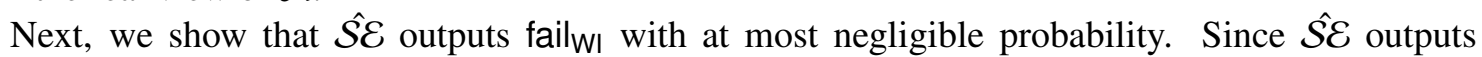
fail ${ }_{W 1}$ when it fails to extract a witness in an accepted right session, this implies that $\hat{\mathcal{S E}}$ extracts a valid witness from every accepted right session except with negligible probability. Assume for contradiction that there exists $\widetilde{i^{*}} \in[\mathrm{m}]$ such that $\hat{\mathcal{S E}}$ outputs failwI during the witness extraction of the $\widetilde{i^{*}}$-th right session with non-negligible probability. Then, let us consider the following hybrid simulator-extractor $\hat{\mathcal{S}} \mathcal{E}_{i^{*}}$.

- $\hat{\mathcal{S E}}{\tilde{i^{*}}}$ is the same as $\hat{\mathcal{S E}}$ except that $\hat{\mathcal{S} \mathcal{E}_{i^{*}}}$ tries to extract a witness only from the $\tilde{i}^{*}$-th right session (and therefore rewinds the wI-main thread only from the challenge message of sWIAOK of the $\widetilde{i}$-th right session). 
Clearly, $\hat{\mathcal{S E}}_{\tilde{i}^{\star}}$ outputs fail ${ }_{\mathrm{WI}}$ with non-negligible probability. Then, we reach a contradiction roughly as follows.

Step 1. First, we show that in $\hat{\mathcal{S} \mathcal{i}_{\tilde{i}^{*}}}$, the probability that $\widetilde{r}_{V}$ is extracted as a witness during the witness extraction of the $\widetilde{i^{*}}$-th right session is non-negligible, where $\widetilde{r}_{V}$ is the value chosen by the verifier in Stage I- 1 of the $\widetilde{i^{*}}$-th right session.

Step 2. Next, we define a sequence of hybrid simulator-extractors, where the first hybrid is the same as $\hat{\mathcal{S}}{\tilde{i^{*}}}_{\widetilde{*}}$, and we gradually modify the $\widetilde{i^{*}}$-th right session so that it is independent of $\widetilde{r}_{V}$ in the last hybrid.

Step 3. Finally, we show that even in the last hybrid, the probability that $\widetilde{r}_{V}$ is extracted during the witness extraction of the $\widetilde{i^{*}}$-th right session is non-negligible. Since the $\widetilde{i^{*}}$-th right session is independent of $\widetilde{r}_{V}$ in the last hybrid, we reach a contradiction.

Details are given below.

Step 1. Prove that $\hat{\mathcal{S}} \varepsilon_{\tilde{i}^{*}}$ extracts $\widetilde{r}_{V}$. We first prove the following claim.

Claim 3. Let $\widetilde{r}_{V}$ be the value chosen by the verifier in Stage I-1 of the $\widetilde{i^{*}}$-th right session. If $\hat{\mathcal{S E}}_{\tilde{i}^{*}}$ outputs fail ${ }_{\mathrm{WI}}$ with non-negligible probability, then in $\hat{\mathcal{S E}}_{\tilde{i}^{*}}$ the probability that $\widetilde{r}_{V}$ is extracted during the witness extraction of the $\widetilde{i^{*}}$-th right session is non-negligible.

Proof. Assume for contradiction that $\widetilde{r}_{V}$ is extracted during the witness extraction of the $\widetilde{i}^{*}$-th right session with at most negligible probability. Then, since we assume that $\hat{\mathcal{S}} \mathcal{E}_{\tilde{i}^{*}}$ outputs fail ${ }_{W I}$ with non-negligible probability, the following occurs in $\hat{\mathcal{S}} \mathcal{E}_{i^{*}}$ with non-negligible probability:

- $\hat{\mathcal{S E}}_{\tilde{i}^{*}}$ obtains two accepting transcript of the $\tilde{i}^{*}$-th right session (and therefore that of sWIAOK) such that the commit-messages of sWIAOK are the same, ${ }^{8}$ but

- from these two transcript, $\hat{\mathcal{S}} \mathcal{E}_{\tilde{i}^{*}}$ fails to extract any witness from sWIAOK (i.e., a witness for $\widetilde{x}_{\tilde{i}^{*}} \in L$ or a valid decommitment of the Stage I-1 commitment).

We first show that when the above occurs, the two accepting sWIAOK transcripts are admissible except with negligible probability. (Recall that a pair of accepted transcripts of sWIAOK are admissible if their commit-messages are the same but their challenge-messages are different.) Toward this end, it suffices to show that $\hat{\mathcal{S} \mathcal{E}_{\tilde{i}^{\star}}}$ chooses the same challenge-message of sWIAOK on the wI-main thread and a wI-auxiliary thread with at most negligible probability. This can be shown as follows.

- From a standard argument, we can show that the expected number of rewinding of the wI-main

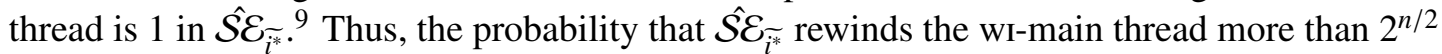
times is at most $2^{-n / 2}$. Furthermore, under the condition that $\hat{\mathcal{S}}_{\tilde{i}^{\star}}$ rewinds the wI-main thread at most $2^{n / 2}$ times, the probability that $\hat{\mathcal{S} \mathcal{E}_{i^{*}}}$ chooses the same challenge-message on the wI-main thread and a wI-auxiliary thread is at most $2^{n / 2} \cdot 2^{-n}=2^{-n / 2}$. Thus, the probability that $\hat{\mathcal{S E}}_{\tilde{i}^{*}}$ chooses the same challenge-message on the wI-main thread and a wI-auxiliary thread is at most $2^{-n / 2}+2^{-n / 2}=\operatorname{negl}(n)$.

\footnotetext{
${ }^{8}$ Recall that WIPOK consists of three stages: commit, challenge, and response.

${ }^{9}$ See Footnote 7.
} 
Thus, with non-negligible probability $\hat{\mathcal{S} \mathcal{E}_{i^{\star}}}$ obtains two admissible transcripts of sWIAOK from which no witness can be computed.

We then reach a contradiction as follows. Since sWIAOK is a parallel version of Blum's Hamiltoniancycle protocol, if no witness is extracted from two admissible transcripts of sWIAOK, a Com $\mathrm{SH}$ commitment in the commit-messages is decommitted to two different values in the transcripts. Thus, we derive a contradiction by breaking the binding property of Com $\mathrm{SH}_{\mathrm{H}}$ using $\hat{\mathcal{S}}_{\tilde{i}^{\star}}$. A problem is that since $\hat{\mathcal{S} \mathcal{E}_{\tilde{i}^{*}}}$ runs in super-polynomial time, the computational biding property of Com $\mathrm{SH}_{\mathrm{SH}}$ may not hold in $\hat{\mathcal{S}} \mathcal{E}_{\tilde{i}^{*}}$. To overcome this problem, we consider hybrid simulator-extractor $\mathcal{S E}_{\tilde{i}^{\star}}$ that emulates the execution of $\hat{\mathcal{S}}_{\widetilde{i}^{\star}}$ in polynomial time. Specifically, $\mathcal{S} \mathcal{E}_{\tilde{i}^{\star}}$ emulates $\hat{\mathcal{S}}_{\tilde{i}^{\star}}$ in the same way as $\mathcal{S} \mathcal{E}$ emu-

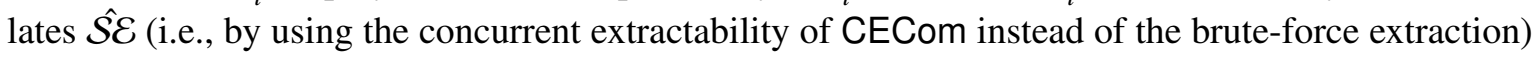
except for the following.

- During the emulation of the wI-main thread, the value $\left(r_{V}, d\right)$ is extracted in Stage I-2 of each left session by using the robust concurrent extractability of CECom so that the commit-message of sWIAOK in the $\tilde{i}^{*}$-th right session is not rewound.

As in the proof of Lemma 2, we can show that $\mathcal{S} \mathcal{E}_{\tilde{i}^{\star}}$ statistically emulates the execution of $\hat{\mathcal{S}} \mathcal{E}_{\tilde{i}^{\star}}$. Thus, with non-negligible probability, $\mathcal{S} \mathcal{E}_{\tilde{i}^{*}}$ obtains two valid decommitments of a $\mathrm{Com}_{\mathrm{SH}}$ commitment (in the commit-messages of sWIAOK of the $\widetilde{i^{*}}$-th right session) such that decommitted values are different. Then, since $\mathcal{S} \mathcal{E}_{\tilde{i}^{*}}$ runs in polynomial time and since the commit-messages of sWIAOK (and therefore the Com $\mathrm{SH}_{\mathrm{H}}$ commitment) of the $\widetilde{i^{*}}$-th right session is not rewound in $\mathcal{S} \mathcal{E}_{\widetilde{i}^{*}}, 10$ we can break the binding property of $\mathrm{Com}_{\mathrm{SH}}$. Thus, we reach a contradiction.

Step 2. Introduce hybrid simulator-extractor. Next, we introduce hybrid simulator-extractors. To clarify the exposition, we first define a sequence of hybrid simulators by gradually modifying $\hat{\mathcal{S}}$ and then define the hybrid simulator-extractors by using them. Below, when we refer to a particular stage of SCNMZK, we always means the corresponding stage of SCNMZK in the $\widetilde{i^{*}}$-th right session.

Hybrid simulator $h-\hat{\mathcal{S}}_{0}$ is identical with $\hat{\mathcal{S}}$.

Hybrid simulator $h-\hat{\mathcal{S}}_{1}$ is the same as $h-\hat{\mathcal{S}}_{0}$ except that $\widetilde{r}_{P}$ is extracted by brute force in Stage II-1 and the committed value of the CECom commitment in Stage II-2 is switched from $0^{n}$ to $\widetilde{r}_{P}$.

Hybrid simulator $h-\hat{\mathcal{S}}_{2}$ is the same as $h-\hat{\mathcal{S}}_{1}$ except that in Stage II-4, the WIPOK proof is computed by using a witness for the fact that the committed value of the CECom commitment in Stage II- 2 is $\widetilde{r}_{P}$.

Hybrid simulator $h-\hat{\mathcal{S}}_{3}$ is the same as $h-\hat{\mathcal{S}}_{2}$ except that in Stage I-2, the committed value of the CECom commitment is switched from $\left(\widetilde{r}_{V}, \widetilde{d}\right)$ to $\left(0^{\widetilde{r}_{V} \mid}, 0^{|\widetilde{d}|}\right)$.

Hybrid simulator $h-\hat{\mathcal{S}}_{4}$ is the same as $h-\hat{\mathcal{S}}_{3}$ except that in Stage I-1, the committed value of the $\mathrm{Com}_{\mathrm{SB}}$ commitment is switched from $\widetilde{r}_{V}$ to $0^{n}$.

Then, for each $k \in\{0, \ldots, 4\}$, hybrid simulator-extractor $h-\hat{\mathcal{S}}_{k}$ is defined as follows.

Hybrid simulator-extractor $h-\hat{\mathcal{S} \mathcal{E}_{k}}$ is the same as $\hat{\mathcal{S}} \mathcal{E}_{\tilde{i}^{*}}$ except that the execution of $\hat{\mathcal{S}}$ is replaced with that of $h-\hat{\mathcal{S}}_{k}$. The output of $h-\hat{\mathcal{S} \mathcal{E}_{k}}$ is the value extracted during the witness extraction of the $\widetilde{i^{*}}$-th right session.

Note that the value $\widetilde{r}_{V}$ is not used anywhere in $h-\hat{\mathcal{S E}}_{4}$.

\footnotetext{
${ }^{10}$ Note that the commit-messages of sWIAOK of the $\widetilde{i^{*}}$-th right session appear only on the wI-main thread.
} 
Step 3. Prove that $\widetilde{r}_{V}$ is extracted in every hybrid. Finally, we show that $\widetilde{r}_{V}$ is extracted with non-negligible probability in each hybrid. First, we consider $h-\hat{\mathcal{S}} \mathcal{E}_{1}$.

Claim 4. Let $\widetilde{r}_{V}$ be the value chosen by the verifier in Stage I-1 of the $\widetilde{i^{*}}$-th right session. If $\hat{\mathcal{S E}}_{\tilde{i}^{*}}$ outputs fail ${ }_{\mathrm{WI}}$ with non-negligible probability, then in $h-\hat{\mathcal{S} \mathcal{E}_{1}}$ the probability that $\widetilde{r}_{V}$ is extracted during the witness extraction of the $\vec{i}^{*}$-th right session is non-negligible.

Proof. In this proof, we use intermediate hybrid simulator-extractors in which the CECom commitment in Stage II-2 of the $\widetilde{i^{*}}$-th right session is gradually modified. Again, we first introduce hybrid simulators. Recall that a CECom commitment consists of $\ell=\omega\left(R_{\mathrm{SH}}(n) \log n\right)$ ExtCom commitments. Then, the intermediate hybrid simulators $h-\hat{\mathcal{S}}_{0: 0}, \ldots, h-\hat{\mathcal{S}}_{0: \ell}$ are defined as follows.

Hybrid simulator $h-\hat{\mathcal{S}}_{0: 0}$ is the same as $h-\hat{\mathcal{S}}_{0}$ except that $\widetilde{r}_{P}$ is extracted by brute force in Stage II-1 of the $\widetilde{i^{*}}$-th right session.

Hybrid simulator $h-\hat{\mathcal{S}}_{0: k}(k \in[\ell])$ is the same as $h$ - $\hat{\mathcal{S}}_{0: k-1}$ except that the committed value of the $k$-th ExtCom commitment in the CECom commitment of Stage II-2 is switched from $0^{n}$ to $\widetilde{r}_{P}$ in the $\widetilde{i^{*}}$-th right session.

Then, for each $k \in\{0, \ldots, \ell\}$, hybrid simulator-extractor $h-\hat{\mathcal{S E}}_{0: k}$ is defined as follows.

Hybrid simulator-extractor $h-\hat{\mathcal{S} \mathcal{E}_{0: k}}$ is the same as $h$ - $\hat{\mathcal{S}}_{0}$ except that the execution of $h-\hat{\mathcal{S}}_{0}$ is replaced with that of $h-\hat{\mathcal{S}}_{0: k}$.

Note that $h-\hat{\mathcal{S}} \mathcal{E}_{0: \ell}$ is identical with $h-\hat{\mathcal{S} \mathcal{E}_{1}}$.

Below, we show that for every $k \in[\ell]$, the output of $h-\hat{\mathcal{S} \mathcal{E}_{0: k-1}}$ and that of $h-\hat{\mathcal{S} \mathcal{E}_{0: k}}$ are indistinguishable. (Recall that the outputs of $h-\hat{\mathcal{S} \mathcal{E}_{0: k-1}}$ and $h-\hat{\mathcal{S} \mathcal{E}_{0: k}}$ are the value extracted in the $\tilde{i}^{*}$-th right session.) Since the probability that $\widetilde{r}_{V}$ is extracted in $h-\hat{\mathcal{S} \mathcal{E}_{0: 0}}$ is non-negligible from Claim 3, this suffices to prove Claim 4.

Roughly speaking, we show this indistinguishability as follows. Since $h-\hat{\mathcal{S}}_{0: k-1}$ and $h-\hat{\mathcal{S}}_{0: k}$ differ only in the committed values of a ExtCom commitment, we use the hiding property of the ExtCom commitment to show the indistinguishability. A problem is that we cannot use it directly since $h-\hat{\mathcal{S} \mathcal{E}_{0: k-1}}$ and $h-\hat{\mathcal{S} \mathcal{E}_{0: k}}$ run in super-polynomial time. To overcome this problem, we observe

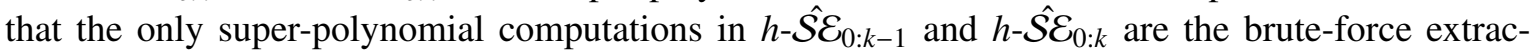
tion of CCACom ${ }^{1: 1}$ (in the $\tilde{i}^{*}$-th right session) and those of CECom (in each left session). Based on this observation, we first show that the execution of $h-\hat{\mathcal{S} \mathcal{E}_{0: k-1}}$ and $h-\hat{\mathcal{S} \mathcal{E}_{0: k}}$ can be emulated in polynomial-time by using the one-session committed-value oracle $O$ of CCACom ${ }^{1: 1}$ and the concurrent extractability of CECom. We then combine the 4-robustness of CCACom ${ }^{1: 1}$ with the hiding property of ExtCom (which has only four rounds) to argue that the output of $h-\hat{\mathcal{S} \varepsilon_{0: k-1}}$ and that of $h-\hat{\mathcal{S} \mathcal{E}_{0: k}}$ are indistinguishable. To formally implement this idea, we need to make sure that the ExtCom commitment and the CCACom ${ }^{1: 1}$ commitment are not rewound during the concurrent extraction of CECom. Details are given below.

First, we introduce hybrid simulator-extractors $h-\mathcal{S} \mathcal{E}_{0: k-1}^{O}$ and $h-\mathcal{S} \mathcal{E}_{0: k}^{O}$, where $O$ is the one-session

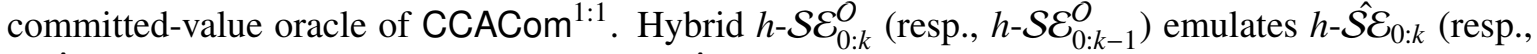
$h-\hat{\mathcal{S} \mathcal{E}_{0: k-1}}$ ) in the same way as $\mathcal{S E}$ emulates $\hat{\mathcal{S} \mathcal{E}}$ except for the following.

- During the emulation of the wI-main thread, the value $\left(r_{V}, d\right)$ is extracted in Stage I-2 of each left session by using the robust concurrent extractability so that the CCACom ${ }^{1: 1}$ commitment in Stage II- 1 and the $k$-th ExtCom commitment in the CECom commitment of Stage II-2 are not rewound in the $\widetilde{i^{*}}$-th right session. In addition, in the $\widetilde{i^{*}}$-th right session, the committed value of $\mathrm{CCACom}^{1: 1}$ is extracted by forwarding the commitment to $O$. Note that the CCACom ${ }^{1: 1}$ commitment in the $\widetilde{i^{*}}$-th right session is not rewound and therefore it can be forwarded to $O$. 
Next, we show that for each $h \in\{k-1, k\}$, the output of $h-\hat{\mathcal{S}}_{0: h}$ and that of $h-\mathcal{S} \mathcal{E}_{0: h}^{O}$ are indistinguishable. This can be proven in a similar way to Lemma 2. In particular, we can use the same argument if we use the following claim instead of Claim 2.

Claim 5. In $h-\hat{\mathcal{S}}_{0: h}$ for each $h \in\{k-1, k\}$, the following holds except with negligible probability: In every left session that reaches Stage III, the CECom commitment in Stage I-2 of this session is valid and its committed value is a valid decommitment of the $\mathrm{Com}_{\mathrm{SB}}$ commitment in Stage I-1.

Claim 5 can be proven in a similar way to Claim 2. For completeness, we give the proof below. (Many texts are taken verbatim from the proof of Claim 2)

Proof of Claim 5. Let us say that a left session is bad if it reaches Stage III and either the CECom commitment in Stage I-2 is invalid or its committed value is not a valid decommitment of the Com $\mathrm{SB}_{\mathrm{B}}$ commitment in Stage I-1; a left session is good if it is not bad. What we want to prove is that every left session is good except with negligible probability.

Roughly speaking, the proof proceeds as follows. From the soundness of WIPOK, if a left session is bad, then in Stage II-2 of this left session, the committed value of the CECom commitment is $r_{P}$, which is the committed value of the CCACom ${ }^{1: 1}$ commitment in Stage II-1; thus, before $r_{P}$ is decommitted to in Stage II-3, we can obtain $r_{P}$ by extracting the committed value from CECom in Stage II-2. This itself does not contradict the hiding property of CCACom ${ }^{1: 1}$ since $h-\hat{\mathcal{S}}_{0: h}$ runs in super-polynomial time in the brute-force extraction of CECom and CCACom ${ }^{1: 1}$. Thus, we again replace the brute-force extraction with the concurrent extraction of CECom and an oracle access to the one-session committed-value oracle $O$ of CCACom ${ }^{1: 1}$, and use the one-one CCA-security of $\mathrm{CCACom}^{1: 1}$ instead of its hiding property. Here, since we want to use the one-one CCA-security of CCACom $^{1: 1}$, we perform the concurrent extraction of CECom so that the CCACom ${ }^{1: 1}$ commitment in a left session and the CCACom ${ }^{1: 1}$ in the $\widetilde{i^{*}}$-th right session are not rewound. Details are given below.

Assume for contradiction that there exists $h \in\{k-1, k\}$ such that in $h$ - $\hat{\mathcal{S}}_{0: h}$, a left session is bad with non-negligible probability. (Here, the indices of the left sessions are determined by the order in which Stage II-3 begins; the reason why we define the indices in this way will become clear later.) Then, there exists $i^{*} \in[m]$ such that in $h-\hat{\mathcal{S}}_{0: h}$, the first $\left(i^{*}-1\right)$ left sessions are good except with negligible probability but the $i^{*}$-th left session is bad with non-negligible probability. Note that from the soundness of WIPOK, when the $i^{*}$-th left session is bad, the committed value of the CECom commitment in Stage II-2 is $r_{P}$ in the $i^{*}$-th left session except with negligible probability, where $r_{P}$ is the value committed to in Stage II-1 of the $i^{*}$-th left session. In the following, we use BAD to denote the event that the $i^{*}$-th left session is bad, and use CHEAT to denote the event that the committed value of the CECom commitment in Stage II-2 is $r_{P}$ in the $i^{*}$-th left session. Then, let us consider the following hybrids.

Hybrid simulator $h-\hat{\mathcal{S}}_{0: h: 0}$ is the same as $h$ - $\hat{\mathcal{S}}_{0: h}$. From our assumption, BAD occurs in $h-\hat{\mathcal{S}}_{0: h: 0}$ with non-negligible probability. Thus, from the above argument, CHEAT occurs in $h-\hat{\mathcal{S}}_{0: h: 0}$ with nonnegligible probability.

Hybrid simulator $h-\hat{\mathcal{S}}_{0: h: 1}$ is the same as $h-\hat{\mathcal{S}}_{0: h: 0}$ except that $h-\hat{\mathcal{S}}_{0: h: 1}$ terminates just before Stage II-3 of the $i^{*}$-th left session begins. Clearly, CHEAT still occurs in $h$ - $\hat{\mathcal{S}}_{0: h: 1}$ with non-negligible probability.

Hybrid simulator $h-\mathcal{S}_{0: h: 1}^{O}$ emulates $h-\hat{\mathcal{S}}_{0: h: 1}$ in polynomial time as follows.

- At the beginning, a random left session $s$ is chosen. (Here, we guess that session $s$ is the $i^{*}$-th left session.) 
- In every left session, in Stage I-2, the committed value $\left(r_{V}, d\right)$ is extracted by the robust concurrent extractor of CECom in such a way that the CCACom ${ }^{1: 1}$ commitment of left session $s$ and the CCACom ${ }^{1: 1}$ commitment of the $\tilde{i}^{*}$-th right session are not rewound. In addition, in the $\widetilde{i^{*}}$-th right session, the committed value of CCACom ${ }^{1: 1}$ is extracted by forwarding the commitment to $O$.

- In left session $s$, the committed value is also extracted in Stage II-2 by the robust concurrent extractor of CECom without rewinding the CCACom ${ }^{1: 1}$ commitment of the $\widetilde{i^{*}}$-th right session.

Note that when Stage III of a left session is executed, the CECom commitment in Stage I-2 of that session is valid except with negligible probability (since that session is one of the first $\left(i^{*}-1\right)$ left sessions and therefore it is good except with negligible probability). Thus, the values extracted from the concurrent extractor are equal to the values that would be extracted by brute force except with negligible probability; therefore, $h-\mathcal{S}_{0: h: 1}^{O}$ statistically emulates $h-\hat{\mathcal{S}}_{0: h: 1}$, and CHEAT occurs in $h-\mathcal{S}_{0: h: 1}^{O}$ with non-negligible probability.

Note that session $s$ is the $i^{*}$-th left session with non-negligible probability. Then, since CHEAT occurs in $h-\mathcal{S}_{0: h: 1}^{O}$ with non-negligible probability, $r_{P}$ is extracted from the CECom commitment in Stage II-2 of session $s$ with non-negligible probability, where $r_{P}$ is the value committed to in Stage II-1 of session $s$. Then, since the CCACom ${ }^{1: 1}$ commitment of session $s$ is not rewound in $h-\mathcal{S}_{0: h: 1}^{O}$, we can break the one-one CCA security of CCACom ${ }^{1: 1}$. Thus, we reach a contradiction.

As argued above, Claim 5 implies that for each $h \in\{k-1, k\}$, the outputs of $h-\hat{\mathcal{S}} \mathcal{E}_{0: h}$ and $h-\mathcal{S} \mathcal{E}_{0: h}^{O}$ are indistinguishable.

To show that the outputs of $h-\hat{\mathcal{S} \mathcal{E}_{0: k-1}}$ and $h-\hat{\mathcal{S} \mathcal{E}_{0: k}}$ are indistinguishable, it remains to prove that the outputs of $h-\mathcal{S} \mathcal{E}_{0: k-1}^{O}$ and $h-\mathcal{S} \mathcal{E}_{0: k}^{O}$ are indistinguishable. This can be shown as follows. Observe that $h-\mathcal{S} \mathcal{E}_{0: k-1}^{O}$ and $h-\mathcal{S} \mathcal{E}_{0: k}^{O}$ differ only in the $k$-th ExtCom commitment of the CECom commitment of the $\widetilde{i}$-th right session, and this ExtCom commitment is not rewound in $h-\mathcal{S} \mathcal{E}_{0: k-1}^{O}$ and $h-\mathcal{S} \mathcal{E}_{0: k}^{O}$. In addition, $h-\mathcal{S} \mathcal{E}_{0: k-1}^{O}$ and $h-\mathcal{S} \mathcal{E}_{0: k}^{O}$ run in polynomial time given oracle access to the one-session committed-value oracle $O$ of CCACom ${ }^{1: 1}$. Thus, from the hiding property of ExtCom and the 4robustness of CCACom ${ }^{1: 1}$, the output of $\mathcal{S} \mathcal{E}_{0: k-1}^{O}$ and that of $h-\mathcal{S} \mathcal{E}_{0: k}^{O}$ are indistinguishable.

Thus, we conclude that the probability that $\widetilde{r}_{V}$ is extracted in $h-\hat{\mathcal{S} \mathcal{E}_{1}}$ is non-negligible. This concludes the proof of Claim 4.

By using essentially the same argument as in the proof of Claim 4, we can show that $\widetilde{r}_{V}$ is extracted with non-negligible probability also in $h-\hat{\mathcal{S E}} \mathcal{E}_{2}, h-\hat{\mathcal{S E}} \mathcal{E}_{3}$, and $h-\hat{\mathcal{S E}}_{4}$. For example, let us consider $h-\hat{\mathcal{S E}}$. Recall that $h-\hat{\mathcal{S}} \mathcal{E}_{2}$ differs from $h-\hat{\mathcal{S} \varepsilon_{1}}$ only in that the different witness is used in WIPOK of the $\widetilde{i^{*}}$-th right session. Then, in the same way as in the proof of Claim 4 , we can define hybrid simulatorextractors $h-\mathcal{S} \mathcal{E}_{1}^{O}$ and $h-\mathcal{S E}_{2}^{O}$ such that the following hold.

- Given oracle access to the one-session committed-value oracle $O$ of CCACom ${ }^{1: 1}$, both $h-\mathcal{S} \mathcal{E}_{1}^{O}$ and $h-\mathcal{S} \mathcal{E}_{2}^{O}$ run in polynomial-time.

- For each $k \in\{1,2\}$, the probability that $\widetilde{r}_{V}$ is extracted in $h-\mathcal{S} \mathcal{E}_{k}^{O}$ is statistically close to the probability in $h-\hat{\mathcal{S E}}_{k}$.

- $h-\mathcal{S} \mathcal{E}_{1}^{O}$ and $h-\mathcal{S} \mathcal{E}_{2}^{O}$ differ only in the witness used in WIPOK of the $\widetilde{i^{*}}$-th right session, and this WIPOK is not rewound in both $h-\mathcal{S} \mathcal{E}_{1}^{O}$ and $h-\mathcal{S} \mathcal{E}_{2}^{O}$. 
Assume for contradiction that $\widetilde{r}_{V}$ is extracted in $h-\hat{\mathcal{S} \mathcal{E}_{2}}$ only with negligible probability. Then, since $\widetilde{r}_{V}$ is extracted in $h-\hat{\mathcal{S E}} \mathcal{E}_{1}$ with non-negligible probability, we can break witness indistinguishability of WIPOK and the 4-robustness of CCACom ${ }^{1: 1}$ by using $h-\mathcal{S} \mathcal{E}_{1}^{O}$ and $h-\mathcal{S} \mathcal{E}_{2}^{O}$. Thus, $\widetilde{r}_{V}$ is extracted in $h-\hat{\mathcal{S E}} \varepsilon_{2}$ with non-negligible probability. In this way, we can show that $\widetilde{r}_{V}$ is extracted also in $h-\hat{\mathcal{S E}} \mathcal{E}_{3}$ and $h-\hat{\mathcal{S E}}_{4}$ with non-negligible probability.

Concluding the proof of Claim 1. In $h-\hat{\mathcal{S E}} \mathcal{E}_{4}$, the $\widetilde{i^{*}}$-th right session is independent of $\widetilde{r}_{V}$, and therefore the probability that $\widetilde{r}_{V}$ is extracted is negligible. However, we show above that this probability is non-negligible. Thus, we reach a contradiction.

This concludes the proof of Theorem 2 .

\section{References}

[BPS06] Boaz Barak, Manoj Prabhakaran, and Amit Sahai. Concurrent non-malleable zero knowledge. In FOCS, pages 345-354, 2006.

[CKPR02] Ran Canetti, Joe Kilian, Erez Petrank, and Alon Rosen. Black-box concurrent zeroknowledge requires (almost) logarithmically many rounds. SIAM J. Comput., 32(1):1-47, 2002.

[CLP10] Ran Canetti, Huijia Lin, and Rafael Pass. Adaptive hardness and composable security in the plain model from standard assumptions. In FOCS, pages 541-550, 2010.

[DDN00] Danny Dolev, Cynthia Dwork, and Moni Naor. Nonmalleable cryptography. SIAM J. Comput., 30(2):391-437, 2000.

[DNS04] Cynthia Dwork, Moni Naor, and Amit Sahai. Concurrent zero-knowledge. J. ACM, 51(6):851-898, 2004

[DPP98] Ivan Damgård, Torben P. Pedersen, and Birgit Pfitzmann. Statistical secrecy and multibit commitments. IEEE Transactions on Information Theory, 44(3):1143-1151, 1998.

[GK96] Oded Goldreich and Ariel Kahan. How to construct constant-round zero-knowledge proof systems for NP. J. Cryptology, 9(3):167-190, 1996.

[GLP ${ }^{+}$15] Vipul Goyal, Huijia Lin, Omkant Pandey, Rafael Pass, and Amit Sahai. Round-efficient concurrently composable secure computation via a robust extraction lemma. In $T C C$, 2015.

[GMOS07] Vipul Goyal, Ryan Moriarty, Rafail Ostrovsky, and Amit Sahai. Concurrent statistical zero-knowledge arguments for NP from one way functions. In ASIACRYPT, pages 444459, 2007.

[HILL99] Johan Håstad, Russell Impagliazzo, Leonid A. Levin, and Michael Luby. A pseudorandom generator from any one-way function. SIAM J. Comput., 28(4):1364-1396, 1999.

[HNO ${ }^{+}$09] Iftach Haitner, Minh-Huyen Nguyen, Shien Jin Ong, Omer Reingold, and Salil P. Vadhan. Statistically hiding commitments and statistical zero-knowledge arguments from any oneway function. SIAM J. Comput., 39(3):1153-1218, 2009. 
[Kiy14] Susumu Kiyoshima. Round-efficient black-box construction of composable multi-party computation. In CRYPTO, pages 351-368, 2014.

[KMO14] Susumu Kiyoshima, Yoshifumi Manabe, and Tatsuaki Okamoto. Constant-round blackbox construction of composable multi-party computation protocol. In TCC, pages 343$367,2014$.

[LP09] Huijia Lin and Rafael Pass. Non-malleability amplification. In STOC, pages 189-198, 2009.

[LP11a] Huijia Lin and Rafael Pass. Concurrent non-malleable zero knowledge with adaptive inputs. In TCC, pages 274-292, 2011.

[LP11b] Huijia Lin and Rafael Pass. Constant-round non-malleable commitments from any oneway function. In STOC, pages 705-714, 2011.

[LP12] Huijia Lin and Rafael Pass. Black-box constructions of composable protocols without set-up. In CRYPTO, pages 461-478, 2012.

[LPTV10] Huijia Lin, Rafael Pass, Wei-Lung Dustin Tseng, and Muthuramakrishnan Venkitasubramaniam. Concurrent non-malleable zero knowledge proofs. In CRYPTO, pages 429-446, 2010.

[LPV08] Huijia Lin, Rafael Pass, and Muthuramakrishnan Venkitasubramaniam. Concurrent nonmalleable commitments from any one-way function. In TCC, pages 571-588, 2008.

[MOSV06] Daniele Micciancio, Shien Jin Ong, Amit Sahai, and Salil P. Vadhan. Concurrent zero knowledge without complexity assumptions. In TCC, pages 1-20, 2006.

[Nao91] Moni Naor. Bit commitment using pseudorandomness. J. Cryptology, 4(2):151-158, 1991.

[NY89] Moni Naor and Moti Yung. Universal one-way hash functions and their cryptographic applications. In STOC, pages 33-43, 1989.

[OOR $\left.{ }^{+} 14\right]$ Claudio Orlandi, Rafail Ostrovsky, Vanishree Rao, Amit Sahai, and Ivan Visconti. Statistical concurrent non-malleable zero knowledge. In TCC, pages 167-191, 2014.

[OPV10] Rafail Ostrovsky, Omkant Pandey, and Ivan Visconti. Efficiency preserving transformations for concurrent non-malleable zero knowledge. In TCC, pages 535-552, 2010.

[PR05] Rafael Pass and Alon Rosen. New and improved constructions of non-malleable cryptographic protocols. In STOC, pages 533-542, 2005.

[PRS02] Manoj Prabhakaran, Alon Rosen, and Amit Sahai. Concurrent zero knowledge with logarithmic round-complexity. In FOCS, pages 366-375, 2002.

[PTV12] Rafael Pass, Wei-Lung Dustin Tseng, and Muthuramakrishnan Venkitasubramaniam. Concurrent zero knowledge, revisited. J. Cryptology, pages 1-22, 2012.

[PW09] Rafael Pass and Hoeteck Wee. Black-box constructions of two-party protocols from oneway functions. In TCC, pages 403-418, 2009.

[Ven14] Muthuramakrishnan Venkitasubramaniam. On adaptively secure protocols. In $S C N$, pages 455-475, 2014. 


\section{A Robust Concurrent Extraction Lemma [GLP ${ }^{+}$15]}

Below, we give a formal description of the robust concurrent extraction lemma [GLP $\left.{ }^{+} 15\right]$.

The external protocol $\Pi$. Let $\Pi:=\langle B, A\rangle$ be an arbitrary two-party computation protocol. For security parameter $n$, let $\operatorname{dom}_{B}(n)$ denote the domain of the input for $B$ and $k:=k(n)$ denote the round complexity of $\Pi$.

The robust-concurrent attack. Let $x \in \operatorname{dom}_{B}(n)$. In the robust-concurrent attack, the adversary $\mathcal{A}$ interacts with a special (possibly super-polynomial-time) party $\mathcal{E}$ called the online extractor. Online extractor $\mathcal{E}$ simultaneously participates in one execution of $\Pi$ and several executions of CECom, where $\mathcal{E}$ interacts with $\mathcal{A}$ as honest $B\left(1^{n}, x\right)$ in the execution of $\Pi$ and interacts with $\mathcal{A}$ as a honest receiver in each execution of CECom. The scheduling of all messages in all sessions- $\Pi$ as well as CECom-is controlled by $\mathcal{A}$. When $\mathcal{A}$ successfully completes a CECom commitment $s$, online extractor $\mathcal{E}$ sends a string $\alpha_{s}$ to $\mathcal{A}$.

For $n \in \mathbb{N}, x \in \operatorname{dom}_{B}(n), z \in\{0,1\}^{*}$, let $\operatorname{REAL}_{\mathcal{E}, \Pi}^{\mathcal{A}}(n, x, z)$ denote the output of the following probabilistic experiment. On input $1^{n}$ and auxiliary input $z$, the experiment starts an execution of $\mathcal{A}$, which launches the robust-concurrent attack by interacting with $\mathcal{E}$. The output of the experiment is the view of $\mathcal{A}$.

The robust concurrent extraction lemma. Informally speaking, the lemma states that there exists an interactive Turing machine-called robust simulator-whose output is statistically close to $\operatorname{REAL}_{\mathcal{E}, \Pi}^{\mathcal{A}}(n, x, z)$ even if the value that the online extractor $\mathcal{E}$ returns to $\mathcal{A}$ at the end of each successful CECom commitment is the committed value of this commitment. Furthermore, the robust simulator does not "rewind" $B$ and runs in time polynomial in total sessions opened by $\mathcal{A}$.

Lemma 3 (Robust Concurrent Extraction Lemma [GLP $\left.\left.{ }^{+} 15\right]\right)$. There exists an interactive Turing machine $\mathcal{S}$ (called robust simulator) such that for every adversary $\mathcal{A}$ and every two-party protocol $\Pi:=\langle B, A\rangle$, there exists a party $\mathcal{E}$ (called online extractor) such that for every $n \in \mathbb{N}, x \in \operatorname{dom}_{B}(n)$, and $z \in\{0,1\}^{*}$, the following conditions hold:

1. Validity constraint. For every output $\rho$ of $\operatorname{REAL}_{\mathcal{E}, \Pi}^{\mathcal{A}}(n, x, z)$ and for every $\mathrm{CECom}$ commitment $s$ appearing in $\rho$, if there exists a unique value $v \in\{0,1\}^{n}$ to which the commitment $s$ can be decommitted, then:

$$
\alpha_{s}=v,
$$

where $\alpha_{s}$ is the value $\mathcal{E}$ sends to $\mathcal{A}$ at the end of $s$.

2. Statistical simulation. Let $k=k(n)$ be the round complexity of $\Pi$. Then the statistical distance between $\operatorname{REAL}_{\mathcal{E}, \Pi}^{\mathcal{A}}(n, x, z)$ and output $\mathcal{S}\left[B\left(1^{n}, x\right) \leftrightarrow \mathcal{S}^{\mathcal{A}}\left(1^{n}, z\right)\right]$ is given by

$$
\Delta(n) \leq 2^{-\Omega(\ell-k \cdot \log T(n))},
$$

where $\ell:=\ell(n)$ is the parameter of CECom and $T(n)$ is the number of the CECom commitments between $\mathcal{A}$ and $\mathcal{E}$. Furthermore, the running time of $\mathcal{S}$ is poly $(n) \cdot T(n)^{2}$. 


\section{B Constant-round One-one CCA-secure Commitment Scheme from OWF}

In this section, we observe that from a result by Goyal et al. [GLP $\left.{ }^{+} 15\right]$, it follows almost immediately that we can obtain a constant-round one-one CCA-secure commitment scheme from one-way functions.

Theorem 3. Assume the existence of one-way functions. Then, for any constant $\kappa \in \mathbb{N}$, there exists $a$ constant-round $\kappa$-robust one-one CCA-secure commitment scheme CCACom ${ }^{1: 1}$.

We use the following building blocks, where all of them can be constructed from one-way functions.

- Constant-round commitment scheme NMCom that is non-malleable w.r.t. itself and any 4-round protocol. Specifically, we use the scheme by Lin and Pass [LP11b]. We remark that, like many other non-malleable commitment schemes, the scheme by [LP11b] also satisfies extractability. ${ }^{11}$ (For the definitions of non-malleability and extractability, see Appendix C.)

- Four-round witness-indistinguishable proof of knowledge WIPOK.

- Constant-round zero-knowledge argument ZKArg [GK96].

- Concurrently extractable commitment scheme CECom of Micciancio et al. [MOSV06] with parameter $\ell=\max \left(\kappa, r_{\mathrm{NM}}, 4\right)+1$, where $r_{\mathrm{NM}}$ is the round complexity of NMCom.

When $\ell=\max \left(\kappa, r_{\mathrm{NM}}, 4\right)+1=O(1)$, CECom does not guarantee concurrent extractability. It is easy to see, however, that it guarantees the following "robust extractability" property: For any adversarial committer $C^{*}$ that commits to a value in a single session of CECom and simultaneously participates an arbitrary $\max \left(\kappa, r_{\mathrm{NM}}, 4\right)$-round protocol $\Pi$, the extractor can extract the committed value from $C^{*}$ without rewinding $\Pi$. For details, see Appendix D.

CCACom $^{1: 1}$ is shown in Figure 6. We remark that CCACom ${ }^{1: 1}$ is almost identical to the CCA-secure commitment scheme of Goyal et al. [GLP $\left.{ }^{+} 15\right]$; essentially, the only difference is the parameter $\ell$ of CECom. We prove its one-one CCA security in Section B.1 and prove its robustness in Section B.2.

\section{B.1 Proof of One-one CCA Security}

For any adversary $\mathcal{A}$ that interacts with the committed-value oracle only in a single session, we show that the following ensembles are computationally indistinguishable.

- $\left\{\operatorname{IND}_{0}(\langle C, R\rangle, \mathcal{A}, n, z)\right\}_{n \in \mathbb{N}, z \in\{0,1\}^{*}}$

- $\left\{\mathrm{IND}_{1}(\langle C, R\rangle, \mathcal{A}, n, z)\right\}_{n \in \mathbb{N}, z \in\{0,1\}^{*}}$

Toward this end, we consider a sequence of hybrid experiments in which the left session of $\operatorname{IND}_{b}(\langle C, R\rangle, \mathcal{A}, n, z)$ is gradually modified so that $\mathcal{A}$ receives no information about $v_{b}$ in the last hybrid.

Hybrid $H_{0}^{b}(n, z)$ is the same as $\operatorname{IND}_{b}(\langle C, R\rangle, \mathcal{A}, n, z)$.

Hybrid $H_{1}^{b}(n, z)$ is the same as $H_{0}^{b}(n, z)$ except for the following.

\footnotetext{
${ }^{11}$ In the scheme of [LP11b], the committer proves by a witness-indistinguishable proof of knowledge system that it knows either the committed value or trapdoor information. Since the scheme is designed so that the trapdoor is hidden from the committer, the committed value can be extracted by extracting the witness from the witness-indistinguishable proof.
} 
Below, CECom is the scheme of Micciancio et al. [MOSV06] with parameter $\ell=$ $\max \left(\kappa, r_{\mathrm{NM}}, 4\right)+1$, where $r_{\mathrm{NM}}$ is the round complexity of NMCom.

\section{Commit Phase}

To commit to $v \in\{0,1\}^{n}$, the committer $C$ does the following with the receiver $R$.

Stage 1. $R$ chooses random $r \in\{0,1\}^{n}$ and commits to $r$ by using CECom. $R$ then prove the validity of this CECom commitment by using ZKArg.

Stage 2. $C$ commits to $v$ by using CECom.

Stage 3. $C$ commits to $0^{n}$ by using NMCom.

Stage 4. $R$ decommits the CECom commitment in Stage 1 to $r$.

Stage 5. $C$ prove the following by using WIPOK:

- the CECom commitment in Stage 2 is valid, or

- the committed value of the NMCom commitment in Stage 3 is $r$.

\section{Decommit Phase}

$C$ decommits the CECom commitment in Stage 2 to $v$.

Figure 6: Constant-round one-one CCA-secure commitment scheme CCACom ${ }^{1: 1}$.

- In Stage 1 of the left session, the committed value $r$ of the CECom commitment is extracted by brute force. If the CECom commitment is invalid or has more then one committed value, $r$ is defined to be a random value.

- In Stage 3 of the left session, the committed value of NMCom is switched from $0^{n}$ to $r$.

Hybrid $H_{2}^{b}(n, z)$ is the same as $H_{1}^{b}(n, z)$ except that in Stage 5 of the left session, the WIPOK proof is computed by using the witness for the fact that the committed value of the NMCom commitment in Stage 3 is $r$. (Notice that from the statistical binding property of CECom, the probability that $\mathcal{A}$ correctly decommits the CECom commitment in Stage 1 to a value other than $r$ is negligible.)

Hybrid $H_{3}^{b}(n, z)$ is the same as $H_{2}^{b}(n, z)$ except that in Stage 2 of the left session, the committed value of CECom is switched from $v_{b}$ to $0^{n}$.

For each $i \in\{0,1,2,3\}$ and $b \in\{0,1\}$, let $\mathrm{H}_{i}^{b}(n, z)$ be the random variable representing the output of $H_{i}^{b}(n, z)$. From the construction, $\mathcal{A}$ receives no information about $v_{b}$ in $H_{3}^{0}(n, z)$ and $H_{3}^{1}(n, z)$ and hence $\mathrm{H}_{3}^{0}(n, z)$ and $\mathrm{H}_{3}^{1}(n, z)$ are identically distributed. Therefore, to show the indistinguishability between the above two ensembles, it suffices to prove that the outputs of each neighboring hybrids are computationally indistinguishable.

Our strategy for proving the indistinguishability of each neighboring hybrids is to reduce their indistinguishability to the security of NMCom, WIPOK, and CECom. The problem of this strategy is the existence of the committed-value oracle: Since the oracle runs in super-polynomial time, the security of NMCom, WIPOK, and CECom might not hold against the adversaries that interact with the oracle. We overcome this problem by showing that the oracle can be emulated efficiently without 
"disturbing" the security of NMCom, WIPOK, and CECom. Specifically, we show that the oracle can be emulated by extracting the committed value of the CECom commitment in Stage 2 of the right session using the extractability of CECom; since CECom provides a robust extractability property, the extraction from CECom does not disturb the security of NMCom, WIPOK, and CECom. We remark that in the formal argument given below, we first show that $\mathcal{A}$ "cheats" in the hybrids only with negligible probability, meaning that in the right session, the committed value of the NMCom commitment in Stage 3 is equal to the committed value of the CECom commitment in Stage 1 only with negligible probability. Showing that $\mathcal{A}$ cheats only with negligible probability is crucial to showing that the oracle can be efficiently emulated. In particular, once we show that $\mathcal{A}$ cheats only with negligible probability, we can use the soundness of WIPOK to argue that the CECom commitment in Stage 2 is valid in the accepted right session except with negligible probability, and thus we can conclude that the extracted value is equal to the committed value when the right session is accepted. The formal argument is given below.

Let us say that $\mathcal{A}$ cheats if the committed value of NMCom in Stage 3 is equal to the committed value $\widetilde{r}$ of CECom in Stage 1 in the accepted right session. First, we show that $\mathcal{A}$ cheats in $H_{0}^{b}(n, z)$ only with negligible probability.

Claim 6. The probability that $\mathcal{A}$ cheats in $H_{0}^{b}(n, z)$ is negligible for each $b \in\{0,1\}$.

Proof. Roughly speaking, this claim follows from the hiding property of CECom-when the adversary cheats, we can obtain $\widetilde{r}$ by extracting the committed value from NMCom, and thus we can obtain the committed value of a CECom commitment before it is decommitted to. To formally implement this idea, it is important that no super-polynomial-time computation is performed during the execution of CECom in Stage 1 of the right session. Fortunately, in $H_{0}^{b}(n, z)$ no super-polynomial-time computation is indeed performed during CECom of the right session, as super-polynomial-time computation is performed only at the end of the right session. (Recall the in the setting of one-one CCA security, $\mathcal{A}$ interacts with the oracle only in a single session.) The formal argument is given below.

Assume for contradiction that there exists $b \in\{0,1\}$ such that $\mathcal{A}$ cheats in $H_{0}^{b}(n, z)$ with nonnegligible probability. Fix any such $b$. To derive a contradiction, we consider the following hybrid experiments.

Hybrid $H_{0: 1}^{b}(n, z)$ is the same as $H_{0}^{b}(n, z)$ except that in Stage 3 of the right session, the committed value of the NMCom commitment is extracted by using the extractability of NMCom.

Clearly, the probability that $\mathcal{A}$ cheats is still non-negligible in $H_{0: 1}^{b}(n, z)$. Hence, from the extractability of NMCom, the extracted value is equal to $\widetilde{r}$ with non-negligible probability.

Hybrid $H_{0: 2}^{b}(n, z)$ is the same as $H_{0: 1}^{b}(n, z)$ except that in Stage 1 of the right session, the ZKArg proof is generated by using the simulator of ZKArg.

From the zero-knowledge property of ZKArg, the probability that $\widetilde{r}$ is extracted from NMCom is still non-negligible in $H_{0: 2}^{b}(n, z)$.

We derive a contradiction by constructing an adversary $\mathcal{B}$ that breaks the hiding property of CECom. Externally, $\mathcal{B}$ interacts with a committer of CECom: It sends random $\widetilde{r}_{0}, \widetilde{r}_{1} \in\{0,1\}^{n}$ to the committer and receives a CECom commitment in which either $\widetilde{r}_{0}$ or $\widetilde{r}_{1}$ is committed. Internally, $\mathcal{B}$ invokes $\mathcal{A}$ and emulates $H_{0: 2}^{b}(n, z)$ for $\mathcal{A}$ honestly except that in Stage 1 of the right session, $\mathcal{B}$ forwards the CECom commitment from the external committer to internal $\mathcal{A}$. Finally, if the value extracted from NMCom is $\widetilde{r}_{1}$ in internally emulated $H_{0: 2}^{b}(n, z), \mathcal{B}$ outputs 1 , and otherwise, it outputs 0 . If $\mathcal{B}$ receives a commitment to $\widetilde{r}_{1}$, it outputs 1 with non-negligible probability from the above argument. On the other hand, if $\mathcal{B}$ receives a commitment to $\widetilde{r}_{0}$, it outputs 1 only with negligible probability since internal $\mathcal{A}$ receives no information about $\widetilde{r}_{1}$. Hence, $\mathcal{B}$ breaks the hiding property of CECom. 
Next, we show that $\mathcal{A}$ cheats only with negligible probability in $H_{1}^{b}(n, z)$, and we use it to prove that $\mathrm{H}_{0}^{b}(n, z)$ and $\mathrm{H}_{1}^{b}(n, z)$ are indistinguishable.

Claim 7. For each $b \in\{0,1\}$, the following hold.

- The probability that $\mathcal{A}$ cheats in $H_{1}^{b}(n, z)$ is negligible.

- $\left\{\mathrm{H}_{0}^{b}(n, z)\right\}_{n \in \mathbb{N}, z \in\{0,1\}^{*}}$ and $\left\{\mathrm{H}_{1}^{b}(n, z)\right\}_{n \in \mathbb{N}, z \in\{0,1\}^{*}}$ are computationally indistinguishable.

Proof. First, we show that $\mathcal{A}$ cheats in $H_{1}^{b}(n, z)$ with negligible probability for each $b \in\{0,1\}$. Roughly speaking, this follows from the non-malleability of NMCom: Since $H_{1}^{b}(n, z)$ differs from $H_{0}^{b}(n, z)$ only in the value committed to in NMCom in the left session, the value that $\mathcal{A}$ commits to by using NMCom in the right session of $H_{1}^{b}(n, z)$ is indistinguishable from the value that $\mathcal{A}$ commits to by using NMCom in the right session of $H_{0}^{b}(n, z)$; hence, from Claim 6, the probability that $\mathcal{A}$ cheats in $H_{1}^{b}(n, z)$ is negligible. We remark that since the left session in $H_{1}^{b}(n, z)$ involves the brute-force extraction of CECom in Stage 1, in the formal argument given below we consider a hybrid experiment in which brute-force extraction is replaced with the rewinding extraction. Since we want to use the non-malleability of NMCom, this extraction is performed in such a way that NMCom in the right session is not rewound. The formal argument is given below.

Assume for contradiction that there exists $b \in\{0,1\}$ such that $\mathcal{A}$ cheats in $H_{1}^{b}(n, z)$ with nonnegligible probability. Fix any such $b$. To derive a contradiction, we consider the following hybrid experiment for $i \in\{0,1\}$.

Hybrid $G_{i}^{b}(n, z)$ is the same as $H_{i}^{b}(n, z)$ except for the following.

- In Stage 1 of the left session, the committed value $r$ of the CECom commitment is extracted by using the extractability of CECom instead of by brute force. Furthermore, this extraction is performed in such a way that the NMCom commitment in the right session is not rewound (see Appendix D).

- $G_{i}^{b}(n, z)$ terminates immediately after NMCom ends in Stage 3 of the right session.

From the soundness of ZKArg, the CECom commitment in Stage 1 of the left session is valid when the ZKArg proof in Stage 1 of the left session is accepted. Hence, when Stage 3 is executed in the left session, the value extracted from the CECom commitment in Stage 1 is equal to its (unique) committed value. Since the only difference from $G_{i}^{b}(n, z)$ and $H_{i}^{b}(n, z)$ is how $r$ is extracted, the view of $\mathcal{A}$ in $G_{i}^{b}(n, z)$ is statistically close to that in $H_{i}^{b}(n, z)$. Therefore, $\mathcal{A}$ cheats in $G_{0}^{b}(n, z)$ with negligible probability from Claim 6 , and $\mathcal{A}$ cheats in $G_{1}^{b}(n, z)$ with non-negligible probability from our hypothesis.

We then derive a contradiction by constructing an adversary $\mathcal{M}$ that breaks the non-malleability of NMCom. Externally, $\mathcal{M}$ interacts with a committer and a receiver of NMCom: It sends $0^{n}$ and $r \in\{0,1\}^{n}$ to the committer and receives a NMCom commitment in which either $0^{n}$ or $r$ is committed to; at the same time, it sends a NMCom commitment to the receiver. Internally, $\mathcal{M}$ invokes $\mathcal{A}$ and emulates $G_{0}^{b}(n, z)$ for $\mathcal{A}$ honestly except for the following.

- After $r$ is extracted in Stage 1 of the left session, $\mathcal{M}$ sends $0^{n}$ and $r$ to the external committer.

- In Stage 3 of the left session, $\mathcal{M}$ forwards the NMCom commitment from the external committer to internal $\mathcal{A}$.

- In Stage 3 of the right session, $\mathcal{M}$ forwards the NMCom commitment from the internal $\mathcal{A}$ to the external receiver. 
From the construction, $\mathcal{M}$ perfectly emulates $G_{0}^{b}(n, z)$ when it receives a NMCom commitment to $0^{n}$, and it perfectly emulates $G_{1}^{b}(n, z)$ when it receives a NMCom commitment to $r$. Hence, when $\mathcal{M}$ receives a NMCom commitment to $0^{n}$, internal $\mathcal{A}$ cheats with negligible probability, and when $\mathcal{M}$ receives a NMCom commitment to $r$, internal $\mathcal{A}$ cheats with non-negligible probability. Then, since the cheating of $\mathcal{A}$ is efficiently recognizable given the view of $\mathcal{M}$ and the committed value of the NMCom commitment in the right session, $\mathcal{M}$ breaks the non-malleability of NMCom.

Next, we show that $\left\{\mathrm{H}_{0}^{b}(n, z)\right\}_{n \in \mathbb{N}, z \in\{0,1\}^{*}}$ and $\left\{\mathrm{H}_{1}^{b}(n, z)\right\}_{n \in \mathbb{N}, z \in\{0,1\}^{*}}$ are computationally indistinguishable. Roughly speaking, this indistinguishability follows from the hiding of NMCom: Since $\mathcal{A}$ cheats only with negligible probability both in $H_{0}^{b}(n, z)$ and in $H_{1}^{b}(n, z)$, the CECom commitment in Stage 2 is valid in the accepted right session in both hybrids; hence the committed-value oracle can be efficiently emulated by extracting the committed value of the CECom commitment in Stage 2, and thus the indistinguishability follows from the hiding property of NMCom. Here, since we want to use the hiding property of NMCom, the extraction from CECom is performed in such a way that NMCom in the left session is not rewound. The formal argument is given below.

Assume for contradiction that there exists $b \in\{0,1\}$ such that $\left\{\mathrm{H}_{0}^{b}(n, z)\right\}_{n \in \mathbb{N}, z \in\{0,1\}^{*}}$ and $\left\{\mathrm{H}_{1}^{b}(n, z)\right\}_{n \in \mathbb{N}, z \in\{0,1\}^{*}}$ are distinguishable. Fix any such $b$. From Claim 6 and what is shown above, $\mathcal{A}$ cheats only with negligible probability both in $H_{0}^{b}(n, z)$ and in $H_{1}^{b}(n, z)$. Hence, from the soundness of WIPOK, the CECom commitment in Stage 2 is invalid in the accepted right session only with negligible probability. Therefore, for infinitely many $n$, there exists $z \in\{0,1\}^{*}$ and a polynomial $p(\cdot)$ such that (i) $\mathrm{H}_{0}^{b}(n, z)$ and $\mathrm{H}_{1}^{b}(n, z)$ are distinguishable with advantage $1 / p(n)$ and (ii) the CECom commitment in Stage 2 of the right session is invalid in the accepted right session with probability at most $1 / 2 p(n)$ in both $H_{0}^{b}(n, z)$ and $H_{1}^{b}(n, z)$. Fix any such $n$ and $z$. From an average argument, there exists a partial transcript $\rho$ of $H_{0}^{b}(n, z)$ up until the end of Stage 1 of the left session such that under the condition that a prefix of the transcript is $\rho$, both of the above (i) and (ii) hold. Let $r$ be the value that is committed to in Stage 1 of the left session in $\rho$. (If the committed value is not uniquely determined, $r$ is a random value.) We consider the following two cases.

Case 1. Stage 2 of the right session has already started in $\rho$. Since the committed value of a CECom commitment is determined by the first message, $\rho$ uniquely determined the committed value $\widetilde{v}$ of the CECom commitment in Stage 2 of the right session. Notice that given $\rho, r$, and $\widetilde{v}$ as auxiliary input, $H_{0}^{b}(n, z)$ and $H_{1}^{b}(n, z)$ can be executed from $\rho$ in polynomial time. Hence, we can derive a contradiction by considering an adversary that breaks the hiding property of NMCom by internally emulating $H_{0}^{b}(n, z)$ from $\rho$ and forwarding a NMCom commitment from the external committer (who commits to either $0^{n}$ or $r$ ) to internally emulated $\mathcal{A}$.

Case 2. Stage 2 of the right session starts after $\rho$. We consider the following hybrid experiment.

In Hybrid $F_{i}^{b}(n, z), H_{i}^{b}(n, z)$ is executed from $\rho$ honestly except for the following.

- In the left session, brute-force extraction of $r$ is not performed, and hardwired $r$ is used.

- In Stage 2 of the right session, the committed value $\widetilde{v}$ of the CECom commitment is extracted by using the extractability of CECom in such a way that NMCom in Stage 3 is not rewound in the left session.

- At the end of the right session, the extracted value $\widetilde{v}$ is returned to $\mathcal{A}$ as the committed value of the right session.

From the definition of $\rho$, the CECom commitment in Stage 2 of the right session is invalid in the accepted right session with probability at most $1 / 2 p(n)$. Since the output of $F_{i}^{b}(n, z)$ differs from that of $H_{i}^{b}(n, z)$ only when the correct committed value is not extracted in the accepted right session (which 
occurs with probability at most $1 / 2 p(n)$ from the above), from our hypothesis, the outputs of $F_{0}^{b}(n, z)$ and $F_{1}^{b}(n, z)$ are distinguishable with advantage $1 / 2 p(n)$. Then, since $F_{0}^{b}(n, z)$ and $F_{1}^{b}(n, z)$ differ only in the value committed to in NMCom and since both experiments run in polynomial time, we can derive a contradiction by considering an adversary that internally emulates $F_{0}^{b}(n, z)$ and forwards a NMCom commitment from the external committer to internally emulated $\mathcal{A}$.

In the same way above, we can prove that the outputs of the other neighboring hybrids are also indistinguishable.

Claim 8. For each $b \in\{0,1\}$, the following hold.

- The probability that $\mathcal{A}$ cheats in $H_{2}^{b}(n, z)$ is negligible.

- $\left\{\mathrm{H}_{1}^{b}(n, z)\right\}_{n \in \mathbb{N}, z \in\{0,1\}^{*}}$ and $\left\{\mathrm{H}_{2}^{b}(n, z)\right\}_{n \in \mathbb{N}, z \in\{0,1\}^{*}}$ are computationally indistinguishable.

Proof. This claim can be proven in essentially the same way as Claim 7. First, we can show that $\mathcal{A}$ cheats in $H_{2}^{b}(n, z)$ only with negligible probability by using the same argument except that we use the non-malleability w.r.t. 4-round protocols of NMCom instead of the non-malleability w.r.t. itself. (Recall that $H_{2}^{b}(n, z)$ differs from $H_{1}^{b}(n, z)$ only in the witness used in WIPOK, which has four rounds.) Next, we can show the indistinguishability between $\left\{\mathrm{H}_{1}^{b}(n, z)\right\}_{n \in \mathbb{N}, z \in\{0,1\}^{*}}$ and $\left\{\mathrm{H}_{2}^{b}(n, z)\right\}_{n \in \mathbb{N}, z \in\{0,1\}^{*}}$ by using the same argument except that we use the witness indistinguishability of WIPOK instead of the hiding property of NMCom. We omit the formal proof.

Claim 9. For each $b \in\{0,1\}$, the following hold.

- The probability that $\mathcal{A}$ cheats in $H_{3}^{b}(n, z)$ is negligible.

- $\left\{\mathrm{H}_{2}^{b}(n, z)\right\}_{n \in \mathbb{N}, z \in\{0,1\}^{*}}$ and $\left\{\mathrm{H}_{3}^{b}(n, z)\right\}_{n \in \mathbb{N}, z \in\{0,1\}^{*}}$ are computationally indistinguishable.

Proof. Like Claim 8, this claim can be proven in essentially the same way as Claim 7. We remark however that since the round complexity of CECom is much more than four, we need to consider a sequence of intermediate hybrid experiments in which the committed value of ExtCom in CECom are switched one by one. We omit the formal proof.

This concludes the proof of one-one CCA security.

\section{B.2 Proof of $\kappa$-robustness}

We show that there exists a simulator $\mathcal{S}$ such that for any adversary $\mathcal{A}$ that interacts with the committedvalue oracle only in a single session, and for any $\kappa$-round PPT ITM $B$, the following are computationally indistinguishable:

- $\left\{\text { output }_{B, \mathcal{A} O}\left[B\left(1^{n}, x, y\right) \leftrightarrow \mathcal{A}^{O}\left(1^{n}, x, z\right)\right]\right\}_{n \in \mathbb{N}, x, y, z \in\{0,1\}^{n}}$

- $\left\{\text { output }_{B, \mathcal{S}^{\mathcal{A}}}\left[B\left(1^{n}, x, y\right) \leftrightarrow \mathcal{S}^{\mathcal{A}}\left(1^{n}, x, z\right)\right]\right\}_{n \in \mathbb{N}, x, y, z \in\{0,1\}^{n}}$

This can be shown easily by using the argument we used in the proof of one-one CCA security. Roughly, we consider a simulator that emulates $O$ for $\mathcal{A}$ efficiently by extracting the committed value of the CECom commitment in Stage 2 using the robust extractability of CECom in such a way that the interaction with $B$ is not rewound. (Since we set $\ell=\max \left(\kappa, r_{\mathrm{NM}}, 4\right)+1$, such extraction is possible.) To show that this simulator indeed emulates the oracle for $\mathcal{A}$, we need to show that the CECom commitment in Stage 2 is invalid in the accepted right session only with negligible probability. This can be shown by using the argument in the proof of Claim 6 . Hence, by using this simulator, we can prove the $\kappa$-robustness. The formal proof is omitted. 


\section{Additional Definitions}

In this section, we give the definitions that are used in Appendix B.

\section{C.1 Non-malleable Commitment Schemes}

\section{Non-malleability w.r.t. Itself}

We recall the definition of non-malleable commitment schemes from [LPV08]. For convenience, we use a slightly different presentation (based on indistinguishability rather than simulation), which is used in [LP09, LP11a]. Let $\langle C, R\rangle$ be a tag-based commitment scheme. For any man-in-the-middle adversary $\mathcal{M}$, consider the following experiment. On input security parameter $n \in \mathbb{N}$ and auxiliary input $z \in\{0,1\}^{*}, \mathcal{M}$ participates in one left and one right interactions simultaneously. In the left interaction, $\mathcal{M}$ interacts with the committer of $\langle C, R\rangle$ and receives a commitment to value $v$ using identity id $\in\{0,1\}^{n}$ of its choice. In the right interaction, $\mathcal{M}$ interacts with the receiver of $\langle C, R\rangle$ and gives a commitment using identity id of its choice. Let $\widetilde{v}$ be the value that $\mathcal{M}$ commits to on the right. If the right commitment is invalid or undefined, $\widetilde{v}$ is defined to be $\perp$. If id $=\widetilde{\mathrm{id}}$, value $\widetilde{v}$ is also defined to be $\perp$. Let $\operatorname{mim}(\langle C, R\rangle, \mathcal{M}, v, z)$ denote a random variable representing $\widetilde{v}$ and the view of $\mathcal{M}$ in the above experiment.

Definition 5. A commitment scheme $\langle C, R\rangle$ is non-malleable if for any PРT man-in-the-middle adversary $\mathcal{M}$, the following are computationally indistinguishable.

- $\{\operatorname{mim}(\langle C, R\rangle, \mathcal{M}, v, z)\}_{n \in \mathbb{N}, v \in\{0,1\}^{n}, v^{\prime} \in\{0,1\}^{n}, z \in\{0,1\}^{*}}$

- $\left\{\operatorname{mim}\left(\langle C, R\rangle, \mathcal{M}, v^{\prime}, z\right)\right\}_{n \in \mathbb{N}, v \in\{0,1\}^{n}, v^{\prime} \in\{0,1\}^{n}, z \in\{0,1\}^{*}}$

\section{Non-malleability w.r.t. $k$-round Protocols}

We recall the definition of non-malleability w.r.t. $\kappa$-round protocols from [LP09]. In [LP09], this property is also referred to as $\kappa$-robustness. We refer to this property as non-malleability w.r.t. $\kappa$ round protocols to distinguish it from the $\kappa$-robustness for CCA secure commitment schemes, which is also used in this work.

Consider a man-in-the-middle adversary $\mathcal{M}$ that participates in a left interaction-communicating with a machine $B$ - and a right interaction - acting as a committer by using the commitment scheme $\langle C, R\rangle$. As in the standard definition of non-malleability, $\mathcal{M}$ can choose the identity in the right interaction. We denote by $\operatorname{mim}(\langle C, R\rangle, B, \mathcal{M}, y, z)$ the random variable consisting of the view of $\mathcal{M}(z)$ in a man-in-the-middle execution when communicating with $B(y)$ on the left and a honest receiver on the right, combined with the values that $\mathcal{M}(z)$ commits to on the right. Intuitively, we say that $\langle C, R\rangle$ is non-malleable w.r.t. $B$ if $\operatorname{mim}\left(\langle C, R\rangle, B, \mathcal{M}, y_{1}, z\right)$ and $\operatorname{mim}\left(\langle C, R\rangle, B, \mathcal{M}, y_{2}, z\right)$ are indistinguishable whenever interactions with $B\left(y_{1}\right)$ and $B\left(y_{2}\right)$ cannot be distinguished.

Definition 6. Let $\langle C, R\rangle$ be a commitment scheme and B be a PPT ITM. We say that the commitment scheme $\langle C, R\rangle$ is non-malleable w.r.t. B if for every two sequences $\left\{y_{n}^{1}\right\}_{n \in \mathbb{N}}$ and $\left\{y_{n}^{2}\right\}_{n \in \mathbb{N}}$ such that for all $\mathrm{PPT}$ ITM $\mathcal{A}$ it holds that

$$
\left\{\operatorname{view}_{\mathcal{A}}\left[B\left(1^{n}, y_{n}^{1}\right) \leftrightarrow \mathcal{A}\left(1^{n}, z\right)\right]\right\}_{n \in \mathbb{N}, z \in\{0,1\}^{*}} \approx\left\{\operatorname{view}_{\mathcal{A}}\left[B\left(1^{n}, y_{n}^{2}\right) \leftrightarrow \mathcal{A}\left(1^{n}, z\right)\right]\right\}_{n \in \mathbb{N}, z \in\{0,1\}^{*}},
$$

it also holds that for every PPT man-in-the-middle adversary $\mathcal{M}$,

$$
\left\{\operatorname{mim}\left(\langle C, R\rangle, B, \mathcal{M}, y_{n}^{1}, z\right)\right\}_{n \in \mathbb{N}, z \in\{0,1\}^{*}} \approx\left\{\operatorname{mim}\left(\langle C, R\rangle, B, \mathcal{M}, y_{n}^{2}, z\right)\right\}_{n \in \mathbb{N}, z \in\{0,1\}^{*}} .
$$

We say that $\langle C, R\rangle$ is non-malleable w.r.t. $\kappa$-round protocols if $\langle C, R\rangle$ is non-malleable w.r.t. any machine $B$ that interacts with the man-in-the-middle adversary in $\kappa$ rounds. 


\section{C.2 Extractable Commitment Scheme}

We recall the definition of extractable commitment schemes from [PW09]. Roughly speaking, a commitment scheme is extractable if there exists an expected polynomial-time oracle machine (called an extractor) $E$ such that for any committer $C^{*}$ that generates a commitment, $E^{C^{*}}$ extracts the committed value when the commitment is valid. We note that when the commitment is invalid, $E$ can output an arbitrary garbage value.

Formally, extractable commitment schemes are defined as follows. A commitment scheme $\langle C, R\rangle$ is extractable if there exists an expected polynomial-time extractor $E$ such that for any PPT committer $C^{*}$, extractor $E^{C^{*}}$ outputs a pair $(\tau, \sigma)$ such that

- $\tau$ is identically distributed with the view of $C^{*}$ interacting with honest receiver $R$ in the commit phase.

- If $\tau$ is accepted, then $\sigma \neq \perp$ except with negligible probability.

- If $\sigma \neq \perp$, then it is statistically impossible to decommit $\tau$ to any value other than $\sigma$.

\section{On the Robust Extractability of CECom}

In this section, we observe that for any constant $\kappa \in \mathbb{N}$, CECom with parameter $\ell=\kappa+1$ satisfies the following robust extractability property: For any adversarial committer $C^{*}$ that commits to a value in a single session of CECom and simultaneously participates an arbitrary $\kappa$-round protocol $\Pi$, the extractor can extract the committed value from $C^{*}$ without rewinding $\Pi$. This property is used to obtain constant-round one-one CCA-secure commitment scheme in Appendix B.

Recall that in CECom, the extractable commitment scheme ExtCom of [PW09] is executed $\ell$ times in the following schedule:

1. First, the commit-stage messages of all sessions (of ExtCom) are exchanged in parallel.

2. Subsequently, the challenge-stage message and the reply-stage message of the $i$-th session are exchanged for each $i \in[\ell]$ in sequence.

Let us call the pair of the challenge-stage message and the reply-stage message of a ExtCom commitment a slot. Since the committed value of a ExtCom commitment can be extracted by rewinding the slot and obtaining a new pair of the challenge-stage message and the reply-stage message (see Figure 2 in Section 3.3), the committed value of a CECom commitment can be extracted by rewinding any of the $\ell$ sequential slots.

Consider the following extractor $E$ against any adversarial committer $C^{*}$. Externally, $E$ participates in a $\kappa$-round protocol $\Pi$. Internally, $E$ invokes $C^{*}$ and forwards all messages of $\Pi$ from the external party to internal $C^{*}$ and vice verse; additionally, $E$ interacts with $C^{*}$ in a session of CECom as a honest receiver. (Without loss of generality, we assume that after $C^{*}$ sends a message of $\Pi$ [resp., a message of CECom], $C^{*}$ immediately receives the next message of $\Pi$ [resp., the next message of CECom].) When the session of CECom ends, $E$ extracts the committed value of the session by rewinding $C^{*}$ in a slot that does not "interleave" with any message of $\Pi$ (i.e, a slot such that $C^{*}$ does not exchange any message of $\Pi$ after receiving the challenge message of the slot until it sends the reply-message of the slot; notice that such a slot always exists because there are $\ell=\kappa+1$ sequential slots). Specifically, $E$ continues to rewind such a slot until it obtains a new pair of the challengestage message and the reply-stage message. If $C^{*}$ requires a message of $\Pi$ after being rewound, $E$ cuts off the execution of $C^{*}$ immediately and rewinds $C^{*}$ again. After obtaining a new pair of the 
challenge-stage message and the reply-stage message, it extracts the committed value by using them.

From the construction, $E$ perfectly emulates the view of $C^{*}$ and does not rewind the external protocol П. Also, from the extractability of ExtCom, the extraction fails only with negligible probability. Hence, it remains to show that $E$ runs in (expected) polynomial time. This can be shown easily by using the standard " $p \times 1 / p$ " argument as follows. For any $i \in[\ell]$ and any partial view $\rho_{i}$ of $C^{*}$ from which the $i$-th slot starts, let prefix $\rho_{i}$ be the event that in the execution of $E$, the view of internal $C^{*}$ up until the beginning of the $i$-th slot is $\rho_{i}$. Let $T_{i}$ be the random variable representing the number of rewinding in the $i$-th slot in $E$, and let $p_{\rho_{i}}$ be the probability that under the condition that prefix $_{\rho_{i}}$ occurs, the $i$-th slot is accepting and it does not interleave with any message of $\Pi$. We then have

$$
\mathrm{E}\left[T_{i} \mid \operatorname{prefix}_{\rho_{i}}\right] \leq p_{\rho_{i}} \cdot 1 / p_{\rho_{i}}=1
$$

for any $\rho_{i}$. Thus, we have

$$
\mathrm{E}\left[T_{i}\right]=\sum_{\rho_{i}} \mathrm{E}\left[T_{i} \mid \operatorname{prefix}_{\rho_{i}}\right] \operatorname{Pr}\left[\operatorname{prefix}_{\rho_{i}}\right] \leq \sum_{\rho_{i}} \operatorname{Pr}\left[\operatorname{prefix}_{\rho_{i}}\right] \leq 1 .
$$

Hence, from the linearity of expectation, the expected number of rewinding of $C^{*}$ in the execution of $E$ is at most $\ell$, and thus the expected running time of $E$ can be bounded by a polynomial. 